Article

\title{
Prognostic Inflammatory Index Based on Preoperative Peripheral Blood for Predicting the Prognosis of Colorectal Cancer Patients
}

\author{
Jinming Fu ${ }^{1,+}$, Ji Zhu ${ }^{2,+} \mathbb{D}$, Fenqi Du ${ }^{3}$, Lijie Zhang ${ }^{2}$, Dapeng Li ${ }^{1}$, Hao Huang ${ }^{1}$, Tian Tian ${ }^{1}$, Yupeng Liu ${ }^{1}$, \\ Lei Zhang ${ }^{1}$, Ying Liu ${ }^{1}$, Yuanyuan Zhang ${ }^{1}$, Jing Xu ${ }^{1}$, Shuhan Meng ${ }^{1}$, Chenyang Jia ${ }^{1}, \operatorname{Simin}$ Sun $^{1}$, Xue Li ${ }^{1}$, \\ Liyuan Zhao ${ }^{1}$, Ding Zhang ${ }^{1}$, Lixin Kang ${ }^{1}$, Lijing Gao ${ }^{1}$, Ting Zheng ${ }^{1}$, Sanjun Cai ${ }^{4}$, Yanlong Liu ${ }^{3, *}$ \\ and Yashuang Zhao ${ }^{1, *}$
}

check for updates

Citation: Fu, J.; Zhu, J.; Du, F.; Zhang, L.; Li, D.; Huang, H.; Tian, T.; Liu, Y.; Zhang, L.; Liu, Y.; Zhang, Y.; et al. Prognostic Inflammatory Index Based on Preoperative Peripheral Blood for Predicting the Prognosis of Colorectal Cancer Patients. Cancers 2021, 13, 3. https://dx.doi.org/10.3390/cancers 13010003

Received: 11 November 2020 Accepted: 19 December 2020 Published: 22 December 2020

Publisher's Note: MDPI stays neutral with regard to jurisdictional claims in published maps and institutional affiliations.

Copyright: () 2020 by the authors. Licensee MDPI, Basel, Switzerland. This article is an open access article distributed under the terms and conditions of the Creative Commons Attribution (CC BY) license (https: / / creativecommons.org/ licenses/by/4.0/).
1 Department of Epidemiology, College of Public Health, Harbin Medical University, Harbin 150081, China; Fu_jinming@hrbmu.edu.cn (J.F.); ldroc1987@gmail.com (D.L.); huanghao@hrbmu.edu.cn (H.H.); 102415@hrbmu.edu.cn (T.T.); liuyupeng@wmu.edu.cn (Y.L.); zhanglie@hrbmu.edu.cn (L.Z.); yingliu0531@gmail.com (Y.L.); zhangyuanyuan941012@gmail.com (Y.Z.); xu806663632@gmail.com (J.X.); mengsh@hrbmu.edu.cn (S.M.); jiachenyang1126@hrbmu.edu.cn (C.J.); sunsimin@hrbmu.edu.cn (S.S.); 2018020158@hrbmu.edu.cn (X.L.); 2018020181@hrbmu.edu.cn (L.Z.); ZhangD_95@hrbmu.edu.cn (D.Z.); kanglixin@hrbmu.edu.cn (L.K.); 2020020185@hrbmu.edu.cn (L.G.); zitty@hrbmu.edu.cn (T.Z.)

2 Department of Radiation Oncology, Fudan University Shanghai Cancer Center, Shanghai 200032, China; leoon.zhu@gmail.com (J.Z.); ljzhang15@fudan.edu.cn (L.Z.)

3 Department of Colorectal Surgery, Harbin Medical University Cancer Hospital, Harbin Medical University, Harbin 150081, China; 2019021558@hrbmu.edu.cn

4 Department of Colorectal Surgery, Fudan University Shanghai Cancer Center, Shanghai 200032, China; caisanjun@gmail.com

* Correspondence: 2355@hrbmu.edu.cn (Y.L.); zhaoyashuang@ems.hrbmu.edu.cn (Y.Z.); Tel.: +86-451-8629-8372 (Y.L.); +86-451-8750-2823 (Y.Z.); Fax: +86-451-8666-3760 (Y.L.); +86-451-8750-2885 (Y.Z.)

+ Both authors have made equal contributions to the work.

Simple Summary: Inflammation plays a critical role in the progression of colorectal cancer (CRC). Peripheral blood cell counts could reflect the extent of systemic inflammation and are readily available in clinical practice. The aim of our study was to construct a novel prognostic inflammatory index (PII) by integrating the blood cell counts associated with prognosis and to evaluate and validate the prognostic value of PII in two independent CRC cohorts. Multivariate Cox analyses in the training cohort of 4154 CRC patients indicated that high OS-PII ( $>4.27)$ and high DFS-PII $(>4.47)$ were significantly associated with worse OS (HR: 1.330, $p<0.001$ ) and worse DFS (HR: 1.366, $p<0.001$ ), which has been validated in the external validation cohort of 5161 patients. Both OS-PII and DFS-PII have a stable prognostic performance at various follow-up times, and the nomograms based on OS-PII and DFS-PII achieved good accuracy in personalized survival prediction of patients with CRC.

Abstract: Host inflammation is a critical component of tumor progression and its status can be indicated by peripheral blood cell counts. We aimed to construct a comprehensively prognostic inflammatory index (PII) based on preoperative peripheral blood cell counts and further evaluate its prognostic value for patients with colorectal cancer (CRC). A total of 9315 patients with stage II and III CRC from training and external validation cohorts were included. The PII was constructed by integrating all the peripheral blood cell counts associated with prognosis in the training cohort. Cox analyses were performed to evaluate the association between PII and overall survival (OS) and disease-free survival (DFS). In the training cohort, multivariate Cox analyses indicated that high OS-PII $(>4.27)$ was significantly associated with worse OS (HR: 1.330, 95\% CI: 1.189-1.489, $p<0.001)$; and high DFS-PII (>4.47) was significantly associated with worse DFS (HR: 1.366, 95\% CI: 1.206-1.548, $p<0.001$ ). The prognostic values of both OS-PII and DFS-PII were validated in the external validation cohort. The nomograms achieved good accuracy in predicting both OS and DFS. Time-dependent ROC analyses showed that both OS-PII and DFS-PII have a stable prognostic performance at various follow-up times. The prognostic value of tumor-node-metastasis staging could be enhanced by combining it with either OS-PII or DFS-PII. We 
demonstrated that PIIs are independent prognostic predictors for CRC patients, and the nomograms based on PIIs can be recommended for personalized survival prediction of patients with CRC.

Keywords: colorectal cancer; peripheral blood cell; prognostic inflammatory index; prognosis; nomograms

\section{Introduction}

Globally, colorectal cancer (CRC) ranks second in terms of mortality, with an estimated 881,000 deaths in 2018 [1]. In China, although CRC is the fifth leading cause of cancer death among both men and women, the mortality of CRC has been increasing in the recent decade [2,3]. Radical resection is the most common treatment for CRC patients; however, approximately one-half of patients will experience a recurrence within the first 3 years after surgery [4]. Tumor-node-metastasis (TNM) staging and specific histological features have been identified as prognostic factors for CRC [5-7]. However, patients with equivalent characteristics may have different survival. Various molecular biomarkers have been reported to be associated with clinical outcomes [8-10], but high cost and sophisticated laboratory measurement limit the application in routine clinical practice. These have led to intense interest in exploring new, cheap, and convenient prognostic biomarkers.

It is now recognized that inflammation is a hallmark of cancer and is closely related to tumor progression [11-14]. Host inflammation response can suppress the antitumor function of adaptive immunity and break the balance between the immune system and malignant tumors, thereby causing the poor prognosis of patients [15]. The inflammatory process frequently causes changes in numerous hematological parameters, such as peripheral blood cell counts and levels of C-reactive protein and albumin. In comparison, peripheral blood cell counts are easy to measure, inexpensive, and widely available in routine clinical practice.

Inflammatory markers, including neutrophil-to-lymphocyte ratio (NLR), platelet-tolymphocyte ratio (PLR), lymphocyte-to-monocyte ratio (LMR), systemic immune-inflammation index (SII, platelets $\times$ neutrophils/lymphocytes), eosinophils and basophils, have been investigated for their prognostic roles in CRC patients [16-25]. However, these markers based on a single type or ratio of two or three types of blood cell counts failed to contain all the information of peripheral blood cells for prognosis prediction. Combining NLR, PLR and LMR may provide more information, but this ignores the fact that these markers share reiterative information (lymphocyte is part of both NLR and LMR).

Therefore, we separately assessed the relationship between all the six types of preoperative peripheral blood cell counts and the prognosis of CRC patients and constructed a prognostic inflammatory index (PII) by integrating the blood cell counts associated with prognosis. We evaluated the prognostic value of PII for CRC patients and validated it in an independent CRC cohort. Furthermore, we developed nomograms for personalized survival prediction of patients with $C R C$, which help clinicians to identify high-risk populations and develop treatment strategies.

\section{Results}

\subsection{Patient Characteristics}

A total of 9315 patients with primary CRC from two independent cohorts were included in this study, consisting of 4471 stage II and 4844 stage III patients. The median follow-up time was 70.0 months (interquartile ranges: 50.0-94.0) in the training cohort, with 1274 deaths during this period. The median follow-up time was 24.0 months (interquartile ranges: 12.0-41.0) in the external validation cohort, with 543 deaths during this period. Demographic and clinical characteristics of patients in the training cohort and external validation cohort are summarized in Table 1. 
Table 1. Patients' baseline characteristics in the training and validation cohorts.

\begin{tabular}{|c|c|c|c|}
\hline Demographic or Characteristic & $\begin{array}{l}\text { Training Cohort } \\
\qquad(N=4154)\end{array}$ & $\begin{array}{c}\text { Validation } \\
\text { Cohort } \\
(N=5161)\end{array}$ & $p$ Value \\
\hline Age (year) ${ }^{a}$ & $59.3 \pm 11.65$ & $58.5 \pm 11.95$ & 0.850 \\
\hline$<60$ & $2103(50.6)$ & $2645(51.2)$ & 0.549 \\
\hline$\geq 60$ & $2051(49.4)$ & $2516(48.8)$ & \\
\hline Gender ${ }^{b}$ & & & 0.804 \\
\hline Male & $2454(59.1)$ & $3062(59.3)$ & \\
\hline Female & $1700(40.9)$ & $2099(40.7)$ & \\
\hline BMI $\left(\mathrm{kg} / \mathrm{m}^{2}\right)^{\mathrm{b}}$ & & & - \\
\hline$<24$ & $1828(44.0)$ & - & \\
\hline$\geq 24$ & $1383(33.3)$ & - & \\
\hline Hypertension $^{\mathrm{b}}$ & & & - \\
\hline No & $3554(85.6)$ & - & \\
\hline Yes & $600(14.4)$ & - & \\
\hline Diabetes mellitus ${ }^{\mathrm{b}}$ & & & - \\
\hline No & $3842(92.5)$ & - & \\
\hline Yes & $312(7.5)$ & - & \\
\hline Tumor location $b$ & & & 0.005 \\
\hline Right colon & $899(21.6)$ & $1220(23.7)$ & \\
\hline Transverse colon & $85(2.0)$ & $110(2.1)$ & \\
\hline Left colon & $256(6.2)$ & $362(7.0)$ & \\
\hline Sigmoid colon & $721(17.4)$ & $773(15.0)$ & \\
\hline Rectum & $2193(52.8)$ & $2696(52.2)$ & \\
\hline Tumor diameter ${ }^{b}$ & & & $<0.001$ \\
\hline$<50 \mathrm{~mm}$ & $1640(39.5)$ & $3446(66.8)$ & \\
\hline$\geq 50 \mathrm{~mm}$ & $2359(56.8)$ & $1710(33.1)$ & \\
\hline Pathological classification $\mathrm{b}$ & & & $<0.001$ \\
\hline Prominence & $2740(66.0)$ & $1277(24.7)$ & \\
\hline Infiltration & $268(6.4)$ & $238(4.6)$ & \\
\hline Ulceration & $160(3.9)$ & $3436(66.6)$ & \\
\hline Infiltration and ulceration & $986(23.7)$ & $210(4.1)$ & \\
\hline Differentiation degree $^{b}$ & & & $<0.001$ \\
\hline Well & $331(8.0)$ & $48(0.9)$ & \\
\hline Moderate & $3225(77.6)$ & $3755(72.8)$ & \\
\hline Poor & $598(14.4)$ & $1358(26.3)$ & \\
\hline Histologic classification ${ }^{b}$ & & & $<0.001$ \\
\hline Adenocarcinoma & $3136(75.5)$ & $4342(84.1)$ & \\
\hline $\begin{array}{c}\text { Mucinous adenocarcinoma + signet ring } \\
\text { cell carcinoma }\end{array}$ & $1018(24.5)$ & $819(15.9)$ & \\
\hline TNM staging ${ }^{b}$ & & & $<0.001$ \\
\hline II & $2385(57.4)$ & $2086(40.4)$ & \\
\hline III & $1769(42.6)$ & $3075(59.6)$ & \\
\hline AJCC staging $\mathrm{II}^{\mathrm{b}}$ & & & $<0.001$ \\
\hline IIA & $974(40.8)$ & $1175(56.3)$ & \\
\hline IIB & $180(7.6)$ & $882(42.3)$ & \\
\hline IIC & $1231(51.6)$ & $29(1.4)$ & \\
\hline AJCC staging III ${ }^{b}$ & & & $<0.001$ \\
\hline IIIA & $122(6.9)$ & $271(8.8)$ & \\
\hline IIIB & $752(42.5)$ & $1727(56.2)$ & \\
\hline IIIC & $895(50.6)$ & $1077(35.0)$ & \\
\hline
\end{tabular}


Table 1. Cont.

\begin{tabular}{|c|c|c|c|}
\hline Demographic or Characteristic & $\begin{array}{l}\text { Training Cohort } \\
\qquad(N=4154)\end{array}$ & $\begin{array}{c}\text { Validation } \\
\text { Cohort } \\
(N=5161)\end{array}$ & $p$ Value \\
\hline Tumor invasion $\mathrm{b}$ & & & $<0.001$ \\
\hline $\mathrm{T} 1-\mathrm{T} 3$ & $1847(44.5)$ & $2588(50.1)$ & \\
\hline $\mathrm{T} 4$ & $2307(55.5)$ & $2573(49.9)$ & \\
\hline Lymph nodes involved ${ }^{b}$ & & & $<0.001$ \\
\hline N0 & $2385(57.4)$ & $2086(40.4)$ & \\
\hline $\mathrm{N} 1-\mathrm{N} 2$ & $1769(42.6)$ & 3075 (59.6) & \\
\hline Cancer nodules ${ }^{b}$ & & & $<0.001$ \\
\hline No & $3863(93.0)$ & $4249(82.3)$ & \\
\hline Yes & $291(7.0)$ & $912(17.7)$ & \\
\hline Nerve invasion $\mathrm{b}$ & & & $<0.001$ \\
\hline No & $3836(92.3)$ & $3898(75.5)$ & \\
\hline Yes & $318(7.7)$ & $1263(24.5)$ & \\
\hline Vascular tumor thrombus ${ }^{b}$ & & & $<0.001$ \\
\hline No & 4009 (96.5) & $3622(70.2)$ & \\
\hline Yes & $145(3.5)$ & $1539(29.8)$ & \\
\hline $\mathrm{CEA}^{\mathrm{b}}$ & & & 0.971 \\
\hline$<5 \mathrm{ng} / \mathrm{mL}$ & $2203(53.0)$ & $2832(54.9)$ & \\
\hline$\geq 5 \mathrm{ng} / \mathrm{mL}$ & $1626(39.1)$ & $2087(40.4)$ & \\
\hline CA19-9 b & & & $<0.001$ \\
\hline$<37 \mathrm{U} / \mathrm{mL}$ & $3026(72.8)$ & $3932(76.2)$ & \\
\hline$\geq 37 \mathrm{U} / \mathrm{mL}$ & $621(14.9)$ & $991(19.2)$ & \\
\hline Postoperative chemotherapy $b$ & & & $<0.001$ \\
\hline No & $2413(58.1)$ & $940(18.2)$ & \\
\hline Yes & $1741(41.9)$ & $4221(81.8)$ & \\
\hline Postoperative radiotherapy $\mathrm{b}$ & & & $<0.001$ \\
\hline No & 3969 (95.5) & $4752(92.1)$ & \\
\hline Yes & $185(4.5)$ & 409 (7.9) & \\
\hline Platelet counts $\left(10^{9} / \mathrm{L}\right)^{\mathrm{c}}$ & $247(204-305)$ & $232(189-283)$ & $<0.001$ \\
\hline Neutrophil counts $\left(10^{9} / \mathrm{L}\right)^{\mathrm{c}}$ & $3.77(2.95-4.83)$ & $3.50(2.80-4.50)$ & $<0.001$ \\
\hline Lymphocyte counts $\left(10^{9} / \mathrm{L}\right)^{\mathrm{c}}$ & $1.89(1.50-2.34)$ & $1.70(1.30-2.10)$ & $<0.001$ \\
\hline Monocyte counts $\left(10^{9} / \mathrm{L}\right)^{\mathrm{c}}$ & $0.43(0.33-0.54)$ & $0.40(0.30-0.50)$ & $<0.001$ \\
\hline Eosinophil counts $\left(10^{9} / \mathrm{L}\right)^{\mathrm{c}}$ & $0.12(0.06-0.20)$ & $0.13(0.08-0.22)$ & $<0.001$ \\
\hline Basophil counts $\left(10^{9} / \mathrm{L}\right)^{\mathrm{c}}$ & $0.04(0.02-0.06)$ & $0.02(0.01-0.04)$ & $<0.001$ \\
\hline
\end{tabular}

Abbreviations: BMI, body mass index; AJCC, American Joint Committee on Cancer; Data are presented as ${ }^{\mathrm{a}}$ mean (standard deviation), ${ }^{\mathrm{b}} \mathrm{n}(\%)$ or ${ }^{\mathrm{c}}$ median (interquartile ranges). The validation cohort did not contain BMI and history of hypertension and diabetes. The number of missing values for BMI, tumor diameter, CEA and CA19-9 were 943, 155, 325 and 507, respectively, in the training cohort. The number of missing values for tumor diameter, CEA, and CA19-9 were 5, 242 and 238, respectively, in the validation cohort.

\subsection{Identification of the PII and the Optimal Cut-Off Value}

The results of restricted cubic spline (RCS) regression suggested that platelet, lymphocyte, and eosinophil counts have nonlinear relationships with overall survival (OS) (Figure S1), while platelet and eosinophil counts have nonlinear relationships with diseasefree survival (DFS) (Figure S2). Thus, platelet, lymphocyte, and eosinophil counts were converted into binary variables for further Cox analyses (Figure S3). According to the results of univariate Cox analyses, platelet, lymphocyte, neutrophil, monocyte, and eosinophil counts were associated with OS, whereas only platelet, neutrophil, monocyte, and eosinophil counts were associated with DFS (Table S1). As the types of blood cells associated with OS and DFS were different, we constructed OS-PII and DFS-PII separately. 
The OS-PII was constructed using platelet, lymphocyte, neutrophil, monocyte and eosinophil counts with weights given by the corresponding coefficients from the multivariate Cox model (Table S1): $(1.878 \times$ Platelet $)+(1.370 \times$ Lymphocyte $)+(0.251 \times$ Neutrophil $)+(4.570 \times$ Monocyte $)+(2.094 \times$ Eosinophil $)$. The DFS-PII was constructed using platelet, neutrophil, monocyte, and eosinophil counts with weights given by the corresponding coefficients from the multivariate Cox model (Table S1): $(2.370 \times$ Platelet $)+$ $(0.415 \times$ Neutrophil $)+(2.600 \times$ Monocyte $)+(2.437 \times$ Eosinophil $)$. X-tile 3.6 .1 software was used to determine the optimal cut-off values for OS-PII and DFS-PII, which were 4.27 and 4.47, respectively (Figure S4). Patients were separated into low PII groups (OS-PII $\leq 4.27$; DFS-PII $\leq 4.47$ ) and high PII groups (OS-PII $>4.27$; DFS-PII $>4.47$ ) for further study.

The association of OS-PII and DFS-PII with clinicopathological characteristics within the training cohort is presented in Table S2. OS-PII was associated with age, gender, body mass index (BMI), hypertension, tumor location, tumor diameter, differentiation degree, tumor invasion, and CA19-9 $(p<0.05)$. DFS-PII was associated with differentiation degree and CA19-9 $(p<0.05)$. In the validation cohort, OS-PII was associated with age, gender, tumor location, tumor diameter, differentiation degree, CEA, and CA19-9, while DFS-PII was associated with age, nerve invasion, vascular tumor thrombus, and CA19-9, which were consistent with the findings in the training cohort (Table S3).

\subsection{Prognostic Value of OS-PII and DFS-PII in the Training Cohort}

As the Kaplan-Meier curves show in Figure 1, patients in the high OS-PII and high DFS-PII groups had significantly poorer survival (log-rank test, $p \leq 0.001$ ). The 1-, 3-, 5and 10-year OS and DFS rates of patients in the low OS-PII group were significantly higher than those of patients in the high OS-PII (Tables S4 and S5).

A

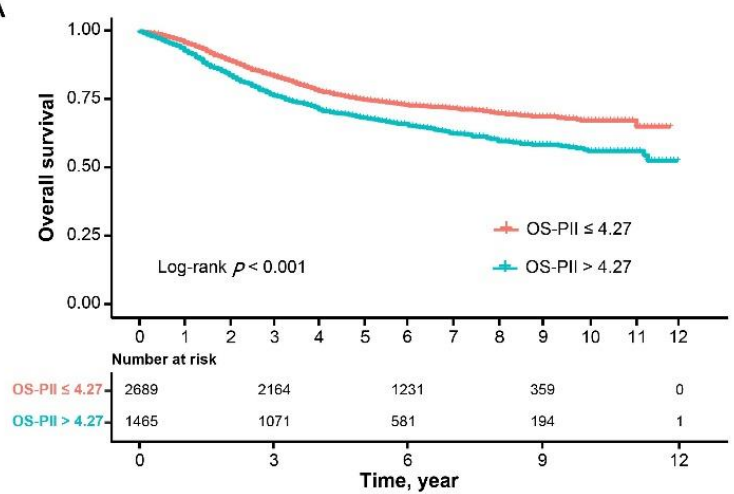

C

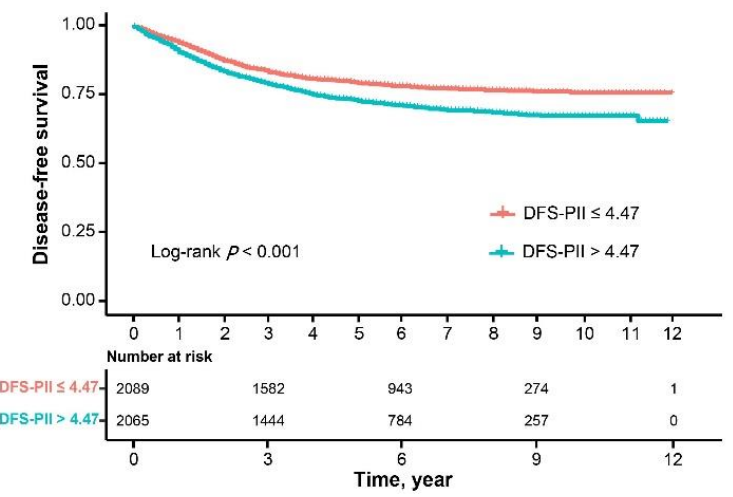

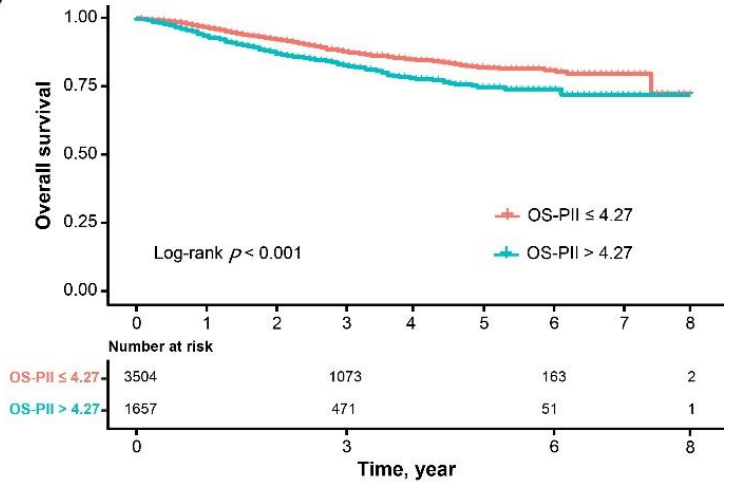

D

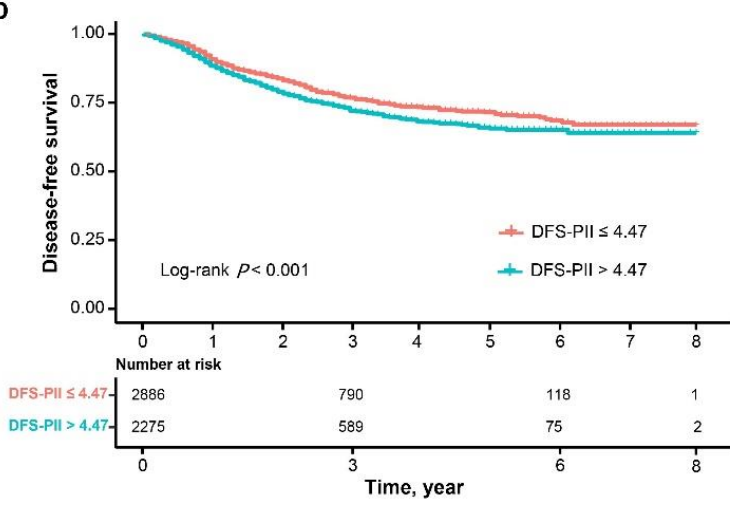

Figure 1. Kaplan-Meier curves of overall survival and disease-free survival for OS-PII and DFS-PII in patients with CRC. Kaplan-Meier curves of overall survival for OS-PII in the training cohort (A), and for OS-PII in the validation cohort (B), and Kaplan-Meier curves of disease-free survival for DFS-PII in the training cohort (C), and for DFS-PII in the validation cohort (D). 
High OS-PII was significantly associated with worse OS in univariate Cox analysis (Table 2). Adjusting for age, gender, tumor location, tumor diameter, pathological classification, differentiation degree, histologic classification, TNM staging, nerve invasion, vascular tumor thrombus, CEA, CA19-9, postoperative chemotherapy and radiotherapy in the multivariate Cox analysis, OS-PII was still statistically associated with the OS of CRC (hazard ratio [HR]: $1.330,95 \%$ confidence interval [CI]: 1.189-1.489, $p<0.001)$, which indicated that OS-PII was an independent prognostic predictor for CRC (Table 2).

Table 2. Association between predictive factors and overall survival of CRC in the training cohort.

\begin{tabular}{|c|c|c|c|c|}
\hline \multirow{2}{*}{ Demographic or Characteristic } & \multicolumn{2}{|c|}{ Univariate Analysis } & \multicolumn{2}{|c|}{ Multivariate Analysis } \\
\hline & HR (95\% CI) & $p$ Value & HR (95\% CI) & $p$ Value \\
\hline Age & & $<0.001$ & & $<0.001$ \\
\hline$<60$ & 1.000 & & 1.000 & \\
\hline$\geq 60$ & $1.654(1.479-1.851)$ & & $1.521(1.354-1.708)$ & \\
\hline Gender & & 0.013 & & $<0.001$ \\
\hline Male & 1.000 & & 1.000 & \\
\hline Female & $0.866(0.773-0.970)$ & & $0.802(0.715-0.899)$ & \\
\hline Tumor location & & $<0.001$ & & 0.001 \\
\hline Colon & 1.000 & & 1.000 & \\
\hline Rectum & $1.297(1.160-1.449)$ & & $1.224(1.089-1.377)$ & \\
\hline Tumor diameter & & $<0.001$ & & 0.009 \\
\hline$<50 \mathrm{~mm}$ & 1.000 & & 1.000 & \\
\hline$\geq 50 \mathrm{~mm}$ & $1.248(1.113-1.400)$ & & $1.171(1.040-1.319)$ & \\
\hline \multicolumn{5}{|l|}{ Pathological classification } \\
\hline Prominence & 1.000 & & 1.000 & \\
\hline Infiltration or Ulceration & $1.544(1.298-1.836)$ & $<0.001$ & $1.357(1.138-1.619)$ & 0.001 \\
\hline Infiltration and Ulceration & $1.578(1.394-1.786)$ & $<0.001$ & $1.394(1.228-1.581)$ & $<0.001$ \\
\hline \multicolumn{5}{|l|}{ Differentiation degree } \\
\hline Well & 1.000 & & 1.000 & \\
\hline Moderate & $1.608(1.243-2.079)$ & $<0.001$ & $1.480(1.142-1.916)$ & 0.003 \\
\hline Poor & $2.812(2.132-3.708)$ & $<0.001$ & $2.269(1.715-3.003)$ & $<0.001$ \\
\hline Histologic classification & & 0.011 & & 0.002 \\
\hline Adenocarcinoma & 1.000 & & 1.000 & \\
\hline $\begin{array}{l}\text { Mucinous adenocarcinoma or signet ring } \\
\text { cell carcinoma }\end{array}$ & $1.176(1.039-1.332)$ & & $1.219(1.073-1.385)$ & \\
\hline TNM staging & & $<0.001$ & & $<0.001$ \\
\hline II & 1.000 & & 1.000 & \\
\hline III & $2.447(2.187-2.738)$ & & $2.248(1.995-2.534)$ & \\
\hline Nerve invasion & & $<0.001$ & & 0.001 \\
\hline No & 1.000 & & 1.000 & \\
\hline Yes & $1.774(1.480-2.126)$ & & $1.387(1.146-1.679)$ & \\
\hline Vascular tumor thrombus & & $<0.001$ & & $<0.001$ \\
\hline No & 1.000 & & 1.000 & \\
\hline Yes & $2.435(1.922-3.087)$ & & $1.669(1.302-2.139)$ & \\
\hline CEA & & $<0.001$ & & $<0.001$ \\
\hline$<5 \mathrm{ng} / \mathrm{mL}$ & 1.000 & & 1.000 & \\
\hline$\geq 5 \mathrm{ng} / \mathrm{mL}$ & $1.709(1.521-1.921)$ & & $1.373(1.217-1.550)$ & \\
\hline CA19-9 & & $<0.001$ & & $<0.001$ \\
\hline$<37 \mathrm{U} / \mathrm{mL}$ & 1.000 & & 1.000 & \\
\hline$\geq 37 \mathrm{U} / \mathrm{mL}$ & $2.012(1.761-2.298)$ & & $1.525(1.322-1.760)$ & \\
\hline
\end{tabular}


Table 2. Cont.

\begin{tabular}{|c|c|c|c|c|}
\hline \multirow{2}{*}{ Demographic or Characteristic } & \multicolumn{2}{|c|}{ Univariate Analysis } & \multicolumn{2}{|c|}{ Multivariate Analysis } \\
\hline & HR $(95 \%$ CI $)$ & $p$ Value & HR $(95 \% \mathrm{CI})$ & $p$ Value \\
\hline Postoperative chemotherapy & & $<0.001$ & & $<0.001$ \\
\hline No & 1.000 & & 1.000 & \\
\hline Yes & $0.669(0.596-0.751)$ & & $0.578(0.511-0.654)$ & \\
\hline Postoperative radiotherapy & & $<0.001$ & & $<0.001$ \\
\hline No & 1.000 & & 1.000 & \\
\hline Yes & $1.854(1.496-2.298)$ & & $1.824(1.458-2.282)$ & \\
\hline OS-PII (Continuous) & $1.105(1.072-1.139)$ & $<0.001$ & $1.087(1.052-1.122)$ & $<0.001$ \\
\hline OS-PII (Binary) & & $<0.001$ & & $<0.001$ \\
\hline$\leq 4.27$ & 1.000 & & 1.000 & \\
\hline$>4.27$ & $1.400(1.253-1.565)$ & & $1.330(1.189-1.489)$ & \\
\hline
\end{tabular}

Abbreviations: HR, hazard ratio; $\mathrm{CI}$, confidence interval.

High DFS-PII was significantly associated with worse DFS in univariate Cox analysis (Table 3). Upon multivariate Cox analysis, DFS-PII was also an independent prognostic predictor for patients with CRC (HR: 1.366, 95\% CI: 1.206-1.548, $p<0.001$ ) (Table 3).

Table 3. Association between predictive factors and disease-free survival of CRC in the training cohort.

\begin{tabular}{|c|c|c|c|c|}
\hline \multirow{2}{*}{ Demographic or Characteristic } & \multicolumn{2}{|c|}{ Univariate Analysis } & \multicolumn{2}{|c|}{ Multivariate Analysis } \\
\hline & HR (95\% CI) & $p$ Value & HR $(95 \%$ CI $)$ & $p$ Value \\
\hline Age (year) & & 0.010 & & $<0.001$ \\
\hline$<60$ & 1.000 & & 1.000 & \\
\hline$\geq 60$ & $1.176(1.040-1.331)$ & & $1.261(1.109-1.433)$ & \\
\hline Gender & & 0.048 & & 0.014 \\
\hline Male & 1.000 & & 1.000 & \\
\hline Female & $0.880(0.776-0.999)$ & & $0.851(0.749-0.968)$ & \\
\hline Tumor location & & $<0.001$ & & $<0.001$ \\
\hline Colon & 1.000 & & 1.000 & \\
\hline Rectum & $1.633(1.437-1.856)$ & & $1.504(1.314-1.721)$ & \\
\hline Tumor diameter & & 0.004 & & 0.008 \\
\hline$<50 \mathrm{~mm}$ & 1.000 & & 1.000 & \\
\hline$\geq 50 \mathrm{~mm}$ & $1.211(1.063-1.379)$ & & $1.195(1.047-1.362)$ & \\
\hline \multicolumn{5}{|l|}{ Pathological classification } \\
\hline Prominence & 1.000 & & 1.000 & \\
\hline Infiltration or Ulceration & $1.559(1.288-1.888)$ & $<0.001$ & $1.395(1.148-1.695)$ & 0.001 \\
\hline Infiltration and Ulceration & $1.498(1.302-1.724)$ & $<0.001$ & $1.316(1.141-1.518)$ & $<0.001$ \\
\hline \multicolumn{5}{|l|}{ Differentiation degree } \\
\hline Well & 1.000 & & 1.000 & \\
\hline Moderate & $1.336(1.029-1.734)$ & 0.030 & 1.257 (0.966-1.637) & 0.088 \\
\hline Poor & $2.161(1.620-2.882)$ & $<0.001$ & $1.708(1.275-2.287)$ & $<0.001$ \\
\hline \multirow{3}{*}{$\begin{array}{c}\text { Histologic classification } \\
\text { Adenocarcinoma } \\
\text { Mucinous adenocarcinoma or signet ring } \\
\text { cell carcinoma }\end{array}$} & & 0.164 & & - \\
\hline & 1.000 & & - & \\
\hline & $1.105(0.960-1.273)$ & & - & \\
\hline TNM staging & & $<0.001$ & & $<0.001$ \\
\hline II & 1.000 & & 1.000 & \\
\hline III & $2.720(2.396-3.088)$ & & $2.148(1.878-2.457)$ & \\
\hline Nerve invasion & & $<0.001$ & & $<0.001$ \\
\hline No & 1.000 & & 1.000 & \\
\hline Yes & $2.084(1.728-2.513)$ & & $1.479(1.212-1.805)$ & \\
\hline
\end{tabular}


Table 3. Cont.

\begin{tabular}{|c|c|c|c|c|}
\hline \multirow{2}{*}{ Demographic or Characteristic } & \multicolumn{2}{|c|}{ Univariate Analysis } & \multicolumn{2}{|c|}{ Multivariate Analysis } \\
\hline & HR $(95 \%$ CI) & $p$ Value & HR $(95 \%$ CI $)$ & $p$ Value \\
\hline Vascular tumor thrombus & & $<0.001$ & & $<0.001$ \\
\hline No & 1.000 & & 1.000 & \\
\hline Yes & $2.810(2.203-3.584)$ & & $1.758(1.361-2.273)$ & \\
\hline CEA & & $<0.001$ & & 0.001 \\
\hline$<5 \mathrm{ng} / \mathrm{mL}$ & 1.000 & & 1.000 & \\
\hline$\geq 5 \mathrm{ng} / \mathrm{mL}$ & $1.530(1.347-1.738)$ & & $1.259(1.100-1.442)$ & \\
\hline CA19-9 & & $<0.001$ & & $<0.001$ \\
\hline$<37 \mathrm{U} / \mathrm{mL}$ & 1.000 & & 1.000 & \\
\hline$\geq 37 \mathrm{U} / \mathrm{mL}$ & $1.914(1.631-2.245)$ & & $1.594(1.353-1.878)$ & \\
\hline Postoperative chemotherapy & & $<0.001$ & & 0.277 \\
\hline No & 1.000 & & 1.000 & \\
\hline Yes & $1.272(1.125-1.439)$ & & $1.076(0.943-1.229)$ & \\
\hline Postoperative radiotherapy & & $<0.001$ & & $<0.001$ \\
\hline No & 1.000 & & 1.000 & \\
\hline Yes & $3.212(2.623-3.932)$ & & $2.281(1.839-2.828)$ & \\
\hline DFS-PII (Continuous) & 1.105 (1.069-1.143) & $<0.001$ & $1.089(1.053-1.128)$ & $<0.001$ \\
\hline DFS-PII (Binary) & & $<0.001$ & & $<0.001$ \\
\hline$\leq 4.47$ & 1.000 & & 1.000 & \\
\hline$>4.47$ & $1.395(1.233-1.580)$ & & $1.366(1.206-1.548)$ & \\
\hline
\end{tabular}

Abbreviations: HR, hazard ratio; CI, confidence interval.

\subsection{Prognostic Value of OS-PII and DFS-PII in the Validation Cohort}

The OS-PII and DFS-PII were further applied to the validation cohort to verify their transportability and generalizability. In the validation cohort, high OS-PII and high DFS-PII correlated significantly with worse OS $(p<0.001)$ and DFS $(p<0.001)$, respectively (Figure 1 ; Tables S6 and S7). In addition, multivariate Cox analysis also revealed that both OS-PII (HR: 1.407, 95\% CI: 1.182-1.674, $p<0.001$ ) and DFS-PII (HR: 1.162, 95\% CI: $1.025-1.318$, $p=0.019)$ were independent prognostic predictors for CRC patients in the validation cohort (Tables 4 and 5). \%clearpage

Table 4. Association between predictive factors and overall survival of CRC in the validation cohort.

\begin{tabular}{|c|c|c|c|c|}
\hline \multirow{2}{*}{ Demographic or Characteristic } & \multicolumn{2}{|c|}{ Univariate Analysis } & \multicolumn{2}{|c|}{ Multivariate Analysis } \\
\hline & HR (95\% CI) & $p$ Value & HR (95\% CI) & $p$ Value \\
\hline Age & & $<0.001$ & & $<0.001$ \\
\hline$<60$ & 1.000 & & 1.000 & \\
\hline$\geq 60$ & $1.418(1.197-1.679)$ & & $1.468(1.235-1.745)$ & \\
\hline Gender & & 0.508 & & 0.604 \\
\hline Male & 1.000 & & 1.000 & \\
\hline Female & $1.059(0.893-1.256)$ & & $1.047(0.881-1.244)$ & \\
\hline Tumor location & & 0.397 & & 0.653 \\
\hline Colon & 1.000 & & 1.000 & \\
\hline Rectum & $0.930(0.786-1.100)$ & & $0.958(0.793-1.156)$ & \\
\hline Tumor diameter & & 0.603 & & 0.288 \\
\hline$<50 \mathrm{~mm}$ & 1.000 & & 1.000 & \\
\hline$\geq 50 \mathrm{~mm}$ & $1.048(0.878-1.252)$ & & $1.106(0.919-1.331)$ & \\
\hline \multicolumn{5}{|l|}{ Pathological classification } \\
\hline Prominence & 1.000 & & 1.000 & \\
\hline Infiltration or Ulceration & $1.488(1.189-1.861)$ & 0.001 & $1.223(0.972-1.539)$ & 0.086 \\
\hline Infiltration and Ulceration & $1.616(1.081-2.415)$ & 0.019 & $1.424(0.948-2.139)$ & 0.088 \\
\hline
\end{tabular}


Table 4. Cont.

\begin{tabular}{|c|c|c|c|c|}
\hline \multirow{2}{*}{ Demographic or Characteristic } & \multicolumn{2}{|c|}{ Univariate Analysis } & \multicolumn{2}{|c|}{ Multivariate Analysis } \\
\hline & HR $(95 \%$ CI) & $p$ Value & HR (95\% CI) & $p$ Value \\
\hline \multicolumn{5}{|l|}{ Differentiation degree } \\
\hline Well & 1.000 & & 1.000 & \\
\hline Moderate & $2.289(0.570-9.196)$ & 0.243 & $1.350(0.334-5.452)$ & 0.673 \\
\hline Poor & $4.904(1.218-19.745)$ & 0.025 & $2.096(0.516-8.513)$ & 0.301 \\
\hline \multirow{3}{*}{$\begin{array}{c}\text { Histologic classification } \\
\text { Adenocarcinoma } \\
\text { Mucinous adenocarcinoma or signet ring } \\
\text { cell carcinoma }\end{array}$} & & 0.001 & & 0.634 \\
\hline & 1.000 & & 1.000 & \\
\hline & $1.413(1.145-1.743)$ & & $1.059(0.838-1.338)$ & \\
\hline TNM staging & & $<0.001$ & & $<0.001$ \\
\hline II & 1.000 & & 1.000 & \\
\hline III & $2.985(2.414-3.691)$ & & $2.083(1.648-2.634)$ & \\
\hline Nerve invasion & & $<0.001$ & & 0.010 \\
\hline No & 1.000 & & 1.000 & \\
\hline Yes & $1.950(1.635-2.325)$ & & $1.284(1.062-1.551)$ & \\
\hline Vascular tumor thrombus & & $<0.001$ & & $<0.001$ \\
\hline No & 1.000 & & 1.000 & \\
\hline Yes & $2.774(2.342-3.285)$ & & $1.721(1.427-2.076)$ & \\
\hline CEA & & $<0.001$ & & $<0.001$ \\
\hline$<5 \mathrm{ng} / \mathrm{mL}$ & 1.000 & & 1.000 & \\
\hline$\geq 5 \mathrm{ng} / \mathrm{mL}$ & $2.375(1.994-2.830)$ & & $1.661(1.376-2.006)$ & \\
\hline CA19-9 & & $<0.001$ & & $<0.001$ \\
\hline$<37 \mathrm{U} / \mathrm{mL}$ & 1.000 & & 1.000 & \\
\hline$\geq 37 \mathrm{U} / \mathrm{mL}$ & $3.073(2.583-3.654)$ & & $1.917(1.581-2.323)$ & \\
\hline Postoperative chemotherapy & & 0.014 & & $<0.001$ \\
\hline No & 1.000 & & 1.000 & \\
\hline Yes & $0.774(0.631-0.949)$ & & $0.618(0.499-0.766)$ & \\
\hline Postoperative radiotherapy & & 0.016 & & 0.089 \\
\hline No & 1.000 & & 1.000 & \\
\hline Yes & $1.329(1.055-1.675)$ & & $1.257(0.965-1.637)$ & \\
\hline OS-PII (Continuous) & $1.164(1.107-1.224)$ & $<0.001$ & $1.133(1.076-1.194)$ & $<0.001$ \\
\hline OS-PII (Binary) & & $<0.001$ & & $<0.001$ \\
\hline$\leq 4.27$ & 1.000 & & 1.000 & \\
\hline$>4.27$ & $1.561(1.316-1.852)$ & & $1.407(1.182-1.674)$ & \\
\hline
\end{tabular}

Abbreviations: $\mathrm{HR}$, hazard ratio; $\mathrm{CI}$, confidence interval.

Table 5. Association between predictive factors and disease-free survival of CRC in the validation cohort.

\begin{tabular}{|c|c|c|c|c|}
\hline \multirow{2}{*}{ Demographic or Characteristic } & \multicolumn{2}{|c|}{ Univariate Analysis } & \multicolumn{2}{|c|}{ Multivariate Analysis } \\
\hline & HR $(95 \%$ CI $)$ & $p$ Value & HR $(95 \%$ CI $)$ & $p$ Value \\
\hline Age (year) & & 0.291 & & 0.108 \\
\hline$<60$ & 1.000 & & 1.000 & \\
\hline$\geq 60$ & $1.070(0.944-1.212)$ & & $1.110(0.978-1.261)$ & \\
\hline Gender & & 0.623 & & 0.328 \\
\hline Male & 1.000 & & 1.000 & \\
\hline Female & $0.969(0.853-1.100)$ & & $0.938(0.825-1.067)$ & \\
\hline Tumor location & & 0.946 & & 0.891 \\
\hline Colon & 1.000 & & 1.000 & \\
\hline Rectum & $1.004(0.888-1.138)$ & & $0.990(0.863-1.136)$ & \\
\hline Tumor diameter & & 0.266 & & 0.951 \\
\hline$<50 \mathrm{~mm}$ & 1.000 & & 1.000 & \\
\hline$\geq 50 \mathrm{~mm}$ & $0.927(0.810-1.060)$ & & $0.996(0.866-1.144)$ & \\
\hline
\end{tabular}


Table 5. Cont.

\begin{tabular}{|c|c|c|c|c|}
\hline \multirow{2}{*}{ Demographic or Characteristic } & \multicolumn{2}{|c|}{ Univariate Analysis } & \multicolumn{2}{|c|}{ Multivariate Analysis } \\
\hline & HR (95\% CI) & $p$ Value & HR $(95 \%$ CI) & $p$ Value \\
\hline \multicolumn{5}{|l|}{ Pathological classification } \\
\hline Prominence & 1.000 & & 1.000 & \\
\hline Infiltration or Ulceration & $1.420(1.208-1.668)$ & $<0.001$ & $1.193(1.013-1.405)$ & 0.035 \\
\hline Infiltration and Ulceration & $1.414(1.040-1.923)$ & 0.027 & $1.232(0.904-1.680)$ & 0.187 \\
\hline \multicolumn{5}{|l|}{ Differentiation degree } \\
\hline Well & 1.000 & & 1.000 & \\
\hline Moderate & $1.796(0.744-4.332)$ & 0.192 & $1.149(0.475-2.780)$ & 0.758 \\
\hline Poor & $2.965(1.225-7.178)$ & 0.016 & $1.435(0.590-3.491)$ & 0.426 \\
\hline \multirow{3}{*}{$\begin{array}{c}\text { Histologic classification } \\
\text { Adenocarcinoma } \\
\text { Mucinous adenocarcinoma or signet ring } \\
\text { cell carcinoma }\end{array}$} & & 0.056 & & - \\
\hline & 1.000 & & - & \\
\hline & $1.174(0.996-1.384)$ & & - & \\
\hline TNM staging & & $<0.001$ & & $<0.001$ \\
\hline II & 1.000 & & 1.000 & \\
\hline III & $2.448(2.112-2.837)$ & & $1.684(1.429-1.984)$ & \\
\hline Nerve invasion & & $<0.001$ & & $<0.001$ \\
\hline No & 1.000 & & 1.000 & \\
\hline Yes & $1.999(1.755-2.277)$ & & $1.389(1.207-1.597)$ & \\
\hline Vascular tumor thrombus & & $<0.001$ & & $<0.001$ \\
\hline No & 1.000 & & 1.000 & \\
\hline Yes & $2.249(1.983-2.551)$ & & $1.528(1.327-1.758)$ & \\
\hline CEA & & $<0.001$ & & $<0.001$ \\
\hline$<5 \mathrm{ng} / \mathrm{mL}$ & 1.000 & & 1.000 & \\
\hline$\geq 5 \mathrm{ng} / \mathrm{mL}$ & $2.004(1.763-2.279)$ & & $1.589(1.384-1.824)$ & \\
\hline CA19-9 & & $<0.001$ & & $<0.001$ \\
\hline$<37 \mathrm{U} / \mathrm{mL}$ & 1.000 & & 1.000 & \\
\hline$\geq 37 \mathrm{U} / \mathrm{mL}$ & $2.306(2.015-2.639)$ & & $1.584(1.364-1.841)$ & \\
\hline Postoperative chemotherapy & & 0.004 & & 0.317 \\
\hline No & 1.000 & & 1.000 & \\
\hline Yes & $1.291(1.087-1.534)$ & & $1.096(0.916-1.312)$ & \\
\hline Postoperative radiotherapy & & 0.001 & & 0.257 \\
\hline No & 1.000 & & 1.000 & \\
\hline Yes & $1.353(1.133-1.615)$ & & $1.121(0.920-1.366)$ & \\
\hline DFS-PII (Continuous) & $1.054(1.036-1.073)$ & 0.003 & $1.037(1.002-1.075)$ & 0.040 \\
\hline DFS-PII (Binary) & & 0.001 & & 0.019 \\
\hline$\leq 4.47$ & 1.000 & & 1.000 & \\
\hline$>4.47$ & $1.248(1.101-1.414)$ & & $1.162(1.025-1.318)$ & \\
\hline
\end{tabular}

Abbreviations: HR, hazard ratio; CI, confidence interval.

\subsection{Prognostic Value of Different Combinations of PIIs and TNM Staging}

The OS and DFS rates of patients with stage II CRC were significantly higher than that of patients with stage III CRC in the training cohort (Tables S4 and S5). In addition, patients in the high OS-PII and high DFS-PII groups had lower OS and DFS rates in both stage II and stage III (Tables S4 and S5). Next, we assessed the association of OS-PII and DFS-PII with prognosis according to different TNM staging.

The stratification by a combination of PIIs and TNM staging divided patients into four risk groups (RG): RG1 (low PIIs and stage II), RG2 (high PIIs and stage II), RG3 (low PIIs and stage III), and RG4 (high PIIs and stage III). Kaplan-Meier curves showed that patients in 
the different RGs demonstrated significantly different survival (Figure 2A,B). Multivariate Cox models adjusting for clinicopathological factors demonstrated that, compared with patients in the RG1 group, the prognosis of patients in the RG2, RG3, and RG4 groups became worse and worse (Figure $2 \mathrm{C}, \mathrm{D}, \mathrm{p}$ for trend $<0.001$ ).

A

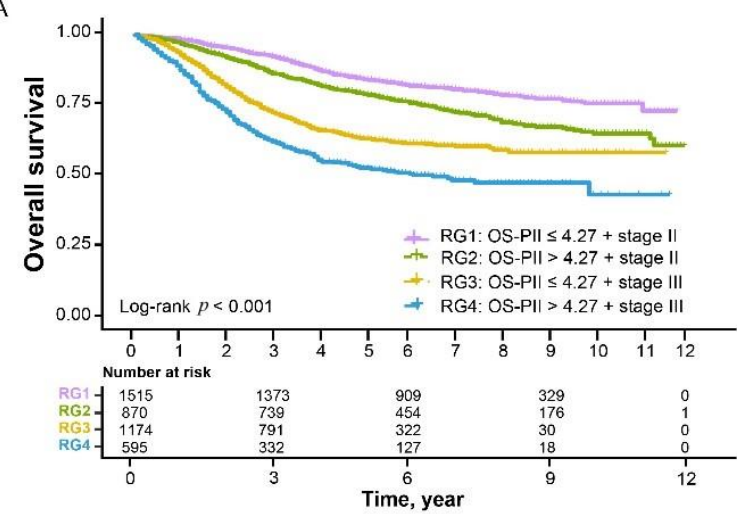

C

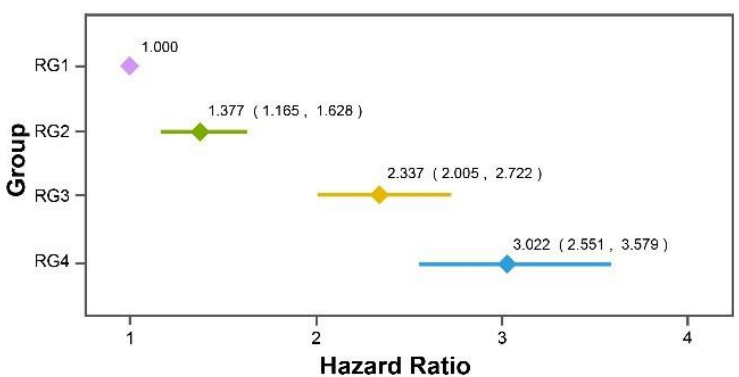

B

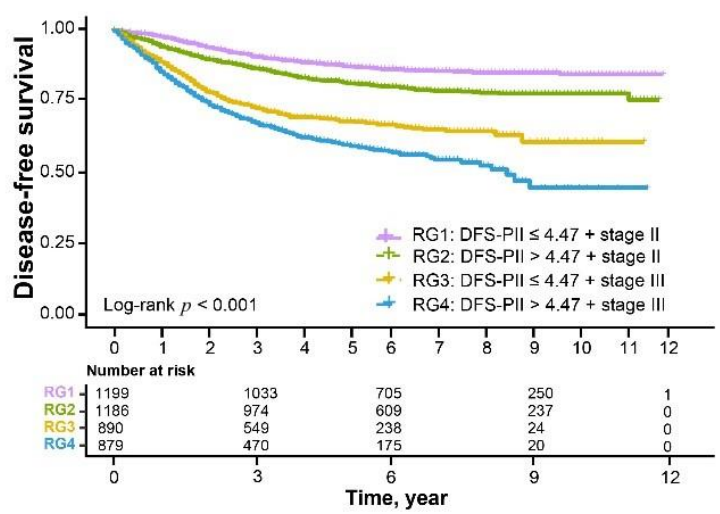

D

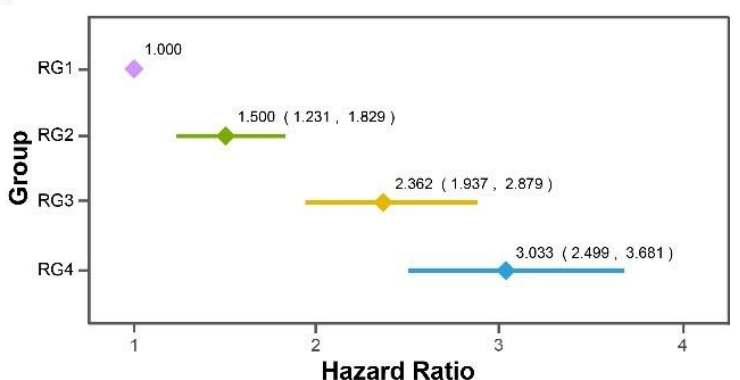

Figure 2. Risk stratification combining PIIs and TNM staging in relation to overall survival and disease-free survival of CRC in the training cohort. Kaplan-Meier curves of four risk groups for overall survival (A) and disease-free survival (B). Multivariate Cox analyses of the four risk groups for overall survival (C) and disease-free survival (D), adjusting for the significant clinicopathological factors in relation to overall survival (Table 2) and disease-free survival (Table 3).

These findings were validated in an external validation cohort, in which patients were also divided into four RGs by a combination of PIIs and TNM staging (Figure S5A,B). After being adjusted for significant clinicopathological factors, patients had worse prognosis as RGs increased (Figure S5C,D, $p$ for trend <0.001).

\subsection{Prognostic Effects of OS-PII and DFS-PII in Different Subgroups}

In the training cohort, the prognostic effects of OS-PII and DFS-PII among different subgroups defined by age, gender, tumor location, tumor diameter, CA19-9, and postoperative chemotherapy (no or yes) were not significantly different (Figure S6). In the validation cohort, the prognostic effects of OS-PII and DFS-PII were also consistent in age $<60$ or $\geq 60$, male or female, colon or rectal cancer, tumor diameter $<50 \mathrm{~mm}$ or $\geq 50 \mathrm{~mm}, \mathrm{CA} 19-9<37 \mathrm{U} / \mathrm{mL}$ or $\geq 37 \mathrm{U} / \mathrm{mL}$, and whether postoperative chemotherapy was taken or not (Figure S7).

\subsection{Comparison of the Prognostic Accuracy of PIIs, TNM Staging, Their Combination, and Previously Reported Biomarkers}

Time-dependent area under the curves (AUCs) associated with OS and DFS were generated to compare the sequential trends of PIIs, TNM staging, their combination, and biomarkers previously reported (NLR, PLR, LMR, and SII). The details of the AUC values of the above markers are listed in Tables S8-S11. In the training cohort, both OS-PII and DFS-PII had a stable prognostic performance at various follow-up times, and their AUCs tended to be higher than the NLR, SII, PLR, and LMR throughout the observation period (Figure 3). The results of tests for comparing the time-dependent AUCs of PIIs with NLR, 
SII, PLR, and LMR showed that both OS-PII and DFS-PII had better accuracy in terms of prognosis prediction (Tables S8 and S9). However, in the validation cohort, time-dependent AUCs among NLR, PLR, LMR, SII, and PIIs did not show significant differences (Tables S10 and S11). Compared with TNM staging alone, the prognostic ability was better with a combination of PIIs and TNM staging. The results were also validated in the external cohort (Figure S8).

A

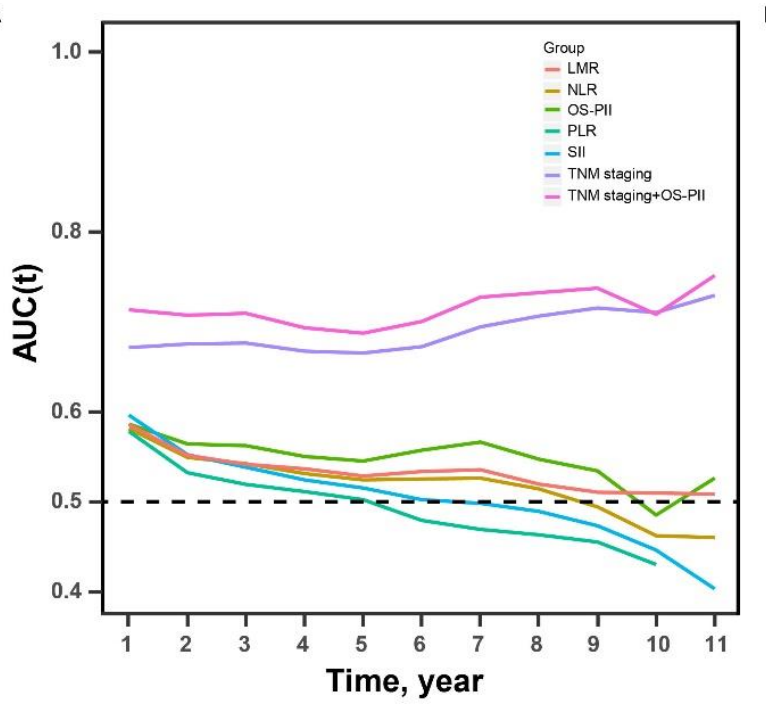

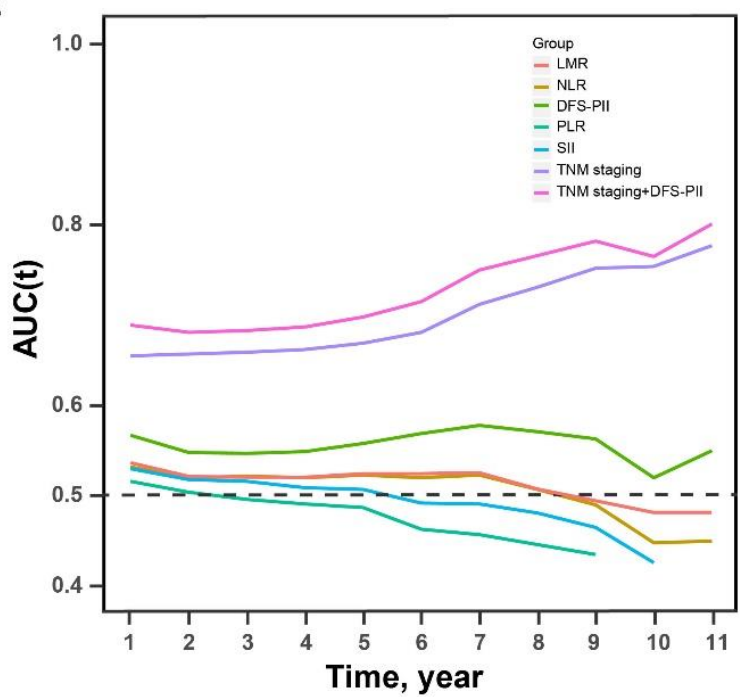

Figure 3. The time-dependent AUCs of PIIs, TNM staging, a combination of PIIs and TNM staging, NLR, PLR, LMR, and SII in the training cohort. Time-dependent AUCs presented the sequential trends of PIIs, TNM staging, a model of PIIs and TNM staging, NLR, PLR, LMR, and SII for overall survival prediction (A) and disease-free survival prediction (B). The horizontal axis represents the years after radical resection, and the vertical axis represents the estimated area under the ROC curves for survival at the time of interest.

\subsection{Development and Validation of Nomograms}

The nomograms based on OS-PII (Figure 4A) and DFS-PII (Figure 5A) were generated for personalized survival prediction of CRC patients. The concordance index (C-index) for OS and DFS prediction were 0.718 (95\% CI: 0.704-0.731) and 0.700 (95\% CI: 0.684-0.716), respectively, in the training cohort, and similar results were observed when we used bootstrapping for internal validation $(0.714$ and 0.694$)$. The C-index for OS and DFS prediction were $0.765(95 \%$ CI: $0.745-0.785)$ and 0.698 (95\% CI: $0.681-0.715)$, respectively, in the validation cohort, and were 0.759 and 0.693 in the internal validation. Compared with AJCC (American Joint Committee on Cancer) system, nomograms had higher C-index (Table S12). The calibration curves for the postoperative 3-year OS (Figure 4B,C) and DFS (Figure 5B,C) showed high agreement between the prediction by nomograms and actual observations. 
A

Points

Tumor location

Tumor diameter

Pathological classification

Differentiation degree

Histologic classification

TNM staging

Nerve invasion

Vascular tumor thrombus

CEA

CA19-9

Postoperative chemotherapy

OS-PII

Total Points

1-year Overall Survival Probability

3-year Overall Survival Probability

5-year Overall Survival Probability

B

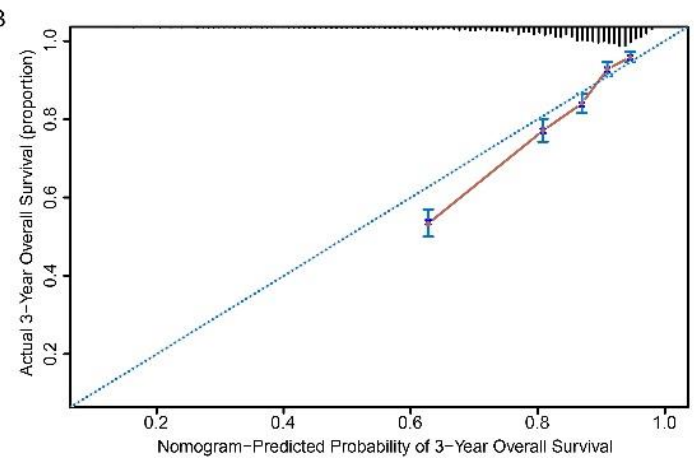

$\leq 4.27$
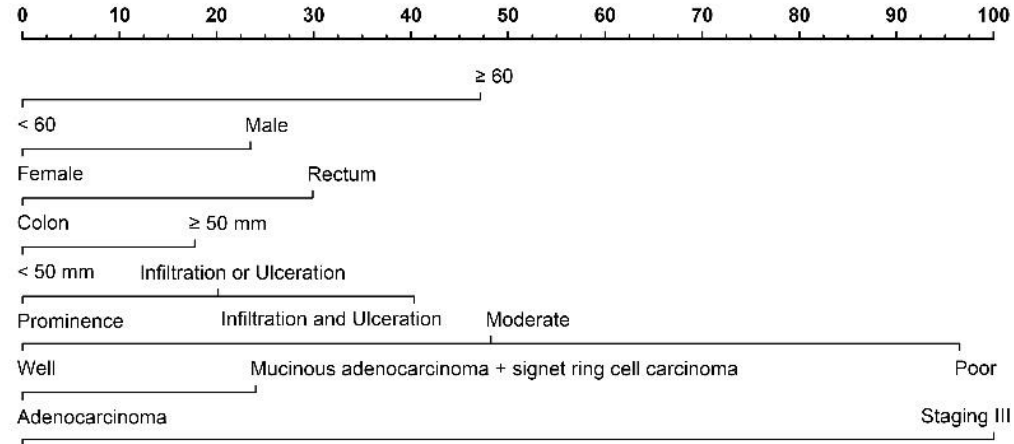

Staging II Yes

No Yes

No $<5 \mathrm{ng} / \mathrm{mL} \quad \geq 5 \mathrm{ng} / \mathrm{mL} \geq 37 \mathrm{U} / \mathrm{mL}$

$<5 \mathrm{ng} / \mathrm{mL}$
$<37 \mathrm{U} / \mathrm{mL}$

Yes $>4.27$

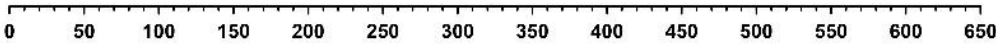

$\begin{array}{lllllll}0.95 & 0.90 & 0.85 & 0.80 & 0.70 & 0.60 & 0.50\end{array}$

\begin{tabular}{lllllllllll}
\hline 0.95 & 0.90 & 0.85 & 0.80 & 0.70 & 0.60 & 0.50 & 0.40 & 0.30 & 0.20 & 0.10
\end{tabular}

$\begin{array}{llllllllll}0.90 & 0.85 & 0.80 & 0.70 & 0.60 & 0.50 & 0.40 & 0.30 & 0.20 & 0.10\end{array}$

C

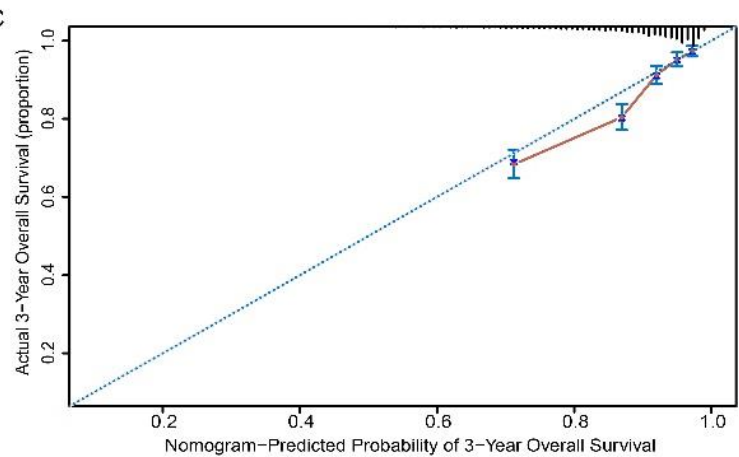

Figure 4. Nomograms to predict overall survival in patients with CRC. Nomograms were performed by using clinicopathological characteristics and OS-PII to predict overall survival (A) and calibration curves of the nomogram to predict overall survival at 3 years in the training cohort (B) and the validation cohort (C). Nomograms map the predicted probabilities onto points on a scale from 0 to 100 and can be interpreted by accumulating the points that correspond to the predicted probability, which is indicated at the top of scale. The total points were converted to predict 1-, 3- and 5-year probabilities of death and recurrence or metastasis for patients with CRC.

\subsection{Decision Curve Analysis}

Decision curve analyses for the prognostic models of the AJCC system and nomograms indicated that both two prognostic models showed a positive net benefit in predicting 5 -year OS and DFS in the training and validation cohorts (Figures S9 and S10). Compared with the AJCC system, the nomograms have better clinical applicability because of their wider range of threshold probabilities and higher net benefit (Figures S9 and S10). The developed nomograms were worth using in terms of personalized survival prediction of patients with CRC. 
A

Points

Age

Gender

Tumor location

Tumor diameter

Pathological classification

Differentiation degree

TNM staging

Nerve invasion

Vascular tumor thrombus

CEA

CA19-9

Postoperative chemotherapy

DFS-PII

Total Points

1-year Disease-free Survival Probability

3-year Disease-free Survival Probability

5-year Disease-free Survival Probability

B

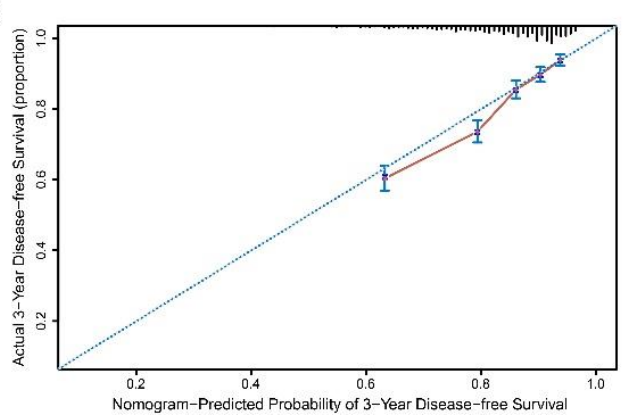

$\leq 4.47$

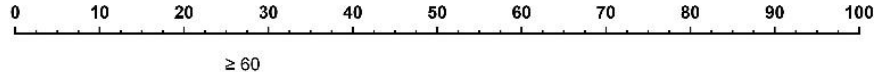

$\geq 60$

\begin{tabular}{ll}
$<60$ & Male \\
\hline Female Rectum & \\
\hline Colon & $\geq 50 \mathrm{~mm}$ \\
$<50 \mathrm{~mm}$ & Infiltration or Ulceration \\
Prominence Moderate Infiltration and Ulceration & Moder
\end{tabular}

Well Poor Staging III

Staging II Yes

No Yes

No $\quad \geq 5 \mathrm{ng} / \mathrm{mL}$

$<5 \mathrm{ng} / \mathrm{mL} \quad \geq 37 \mathrm{U} / \mathrm{mL}$

$<37 \mathrm{U} / \mathrm{mL} \quad$ No

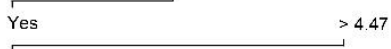

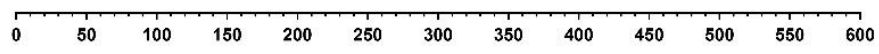
$\begin{array}{lllllllll}0.95 & 0.90 & 0.85 & 0.80 & 0.70 & 0.60 & 0.50 & 0.40 & 0.30\end{array}$

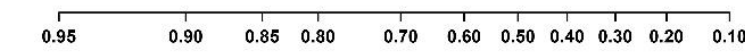

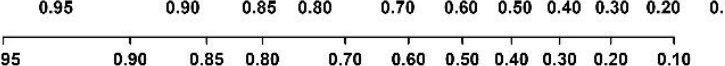

C

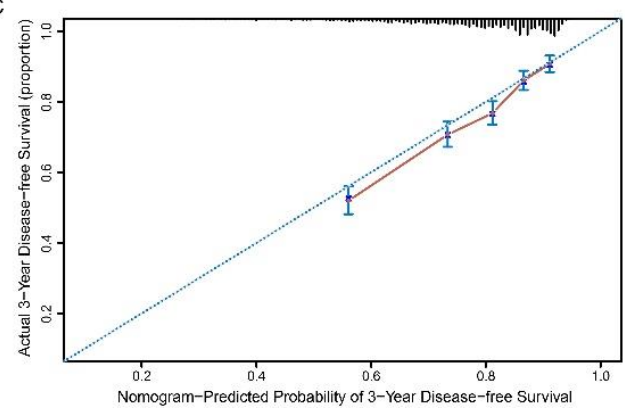

Figure 5. Nomograms to predict disease-free survival in patients with CRC. Nomograms were performed by using clinicopathological characteristics and DFS-PII to predict disease-free survival (A) and calibration curves of the nomogram to predict disease-free survival at 3 years in the training cohort (B) and the validation cohort (C). Nomograms map the predicted probabilities onto points on a scale from 0 to 100 and can be interpreted by accumulating the points that correspond to the predicted probability, which is indicated at the top of scale. The total points were converted to predict 1-, 3- and 5-year probabilities of death and recurrence or metastasis for patients with CRC.

\section{Discussion}

In this double-center, large sample retrospective cohort study, we systemically assessed the prognostic effects of preoperative peripheral blood platelet, neutrophil, lymphocyte, monocyte, eosinophil, and basophil counts in patients with CRC, and constructed a novel inflammatory index by integrating blood cell counts significantly associated with prognosis. Both OS-PII and DFS-PII were independent prognostic predictors for CRC patients and could separate patients into low-risk and high-risk groups. In the prognosis prediction of patients with CRC, both OS-PII and DFS-PII had a stable performance at different time points and could enhance the prognostic ability of TNM staging by combination. Furthermore, the nomograms including PIIs and clinicopathological characteristics could provide personalized OS and DFS prediction and help clinicians to identify high-risk populations. All these findings have been validated in an independent external cohort.

Inflammation, as an important hallmark of cancer [14], plays a critical role in all stages of tumorigenesis $[11,12,15,26,27]$. CRC is inflammation-driven cancer, as is known that inflammatory bowel diseases increase the risk of CRC [14,28]. Peripheral blood cell counts could reflect the extent of systemic inflammation and are readily available in clinical practice. Thus, inflammatory markers constructed based on blood cell counts, have been 
considered as potential prognostic predictors for CRC patients. Studies have mainly been focused on markers such as NLR, PLR, LMR, and SII, with the results showing that elevated NLR, PLR, and SII, and reduced LMR were associated with poor prognosis in CRC [19-22,29-37]. However, these markers were simply based on the ratio of two or three types of blood cell counts, which may not accurately provide information on the status of inflammation. In addition, these markers have not been validated in an external cohort. This context highlights that the development of a comprehensive peripheral blood marker is urgently needed for the identification of patients with CRC with high risk.

Neutrophils, as the first responders to inflammation, can migrate toward the tumor by CXC-chemokines $[38,39]$ and promote the spread of cancer cells to distant organs [40]. Monocytes in the tumor microenvironment have the potential to differentiate into dendritic cells or tumor-associated macrophages [41,42]. Lymphocytes are important components for adaptive immunity and they suppress cancer cell proliferation by inducing apoptosis and inhibiting cancer cell migration and invasion [43]. The effects of platelets [44,45] and eosinophils [46,47] in cancer development and progression are controversial, whereas the effect of basophils on cancer is unknown. However, the relationship between the six types of peripheral blood cells and the prognosis of patients with CRC has not been systematically and clearly elucidated yet. We, for the first time, simultaneously investigated the potential relationship between these six types of blood cells and the prognosis of CRC. The results showed that platelets and eosinophils had a nonlinear relationship with both OS and DFS, which can explain the controversial roles of platelets and eosinophils in cancer; lymphocytes had a nonlinear relationship with OS, but were not associated with DFS, which also indirectly validated the results reported before [48]; and neutrophils and monocytes showed positive linear associations with OS and DFS, but basophils were not associated with the prognosis.

Based on the prognostic effects of different types of peripheral blood cell counts, we constructed the OS-PII and DFS-PII, respectively. The OS-PII and DFS-PII we presented here are innovative because they contained all the types of blood cells associated with prognosis and were integrated based on a weighted approach. Different from most of the published studies that assessed the prognosis of CRC using categorical variables of markers, we found that both continuous and binary variables of the OS-PII and DFS-PII are significant independent prognostic predictors (Tables 2-5). Importantly, the prognostic value of OS-PII and DFS-PII were successfully validated in an independent cohort.

The CRC 5-year relative survival ranges from greater than $90 \%$ in patients with stage I to slightly greater than $10 \%$ in patients with stage IV [49]. Although TNM staging provides valuable prognostic information, the outcome of individual patients is not predicted accurately. This is a drawback for patients with stage II and III CRC in particular but also reminds us of the importance of developing well-performed markers. These significant biomarkers can help identify populations at high risk for recurrence or death in stage II and III patients. We developed the PIIs in a large sample, including patients with stage II and III CRC with sufficient follow-up. After stratification of patients by a combination of PIIs and TNM staging, we found that both OS-PII and DFS-PII had the ability to independently identify high-risk populations in the same TNM staging.

According to the results of time-dependent ROC analyses in the training cohort, the PIIs were superior to NLR, PLR, LMR, and SII in prediction. Unfortunately, this was not validated in the external independent cohort. Considering that the proportion of TNM staging in the two cohorts was inconsistent, time-dependent ROC analyses stratified by TNM staging in both training and validation cohorts were further performed. We found that the time-dependent AUCs showed little difference between stage II and stage III in the training cohort, but the difference became larger in the validation cohort (Tables S13 and S14). The differential proportion of TNM staging in the two cohorts may affect the prediction ability evaluation of PIIs. Collectively, the PIIs were not inferior to reported biomarkers and could be used as a marker for tumor progression and prognosis prediction in patients with CRC. 
Nomograms, which can predict an individual's probability of a clinical event by integrating diverse prognostic variables, are widely used in oncology [50]. Nomograms have been validated to compare favorably to the conventional TNM staging systems in many cancers [51,52]. Our study has built nomograms that assign predictions for survival based on both PIIs and significant clinicopathological factors. The nomograms performed well for predicting both OS (C-index: 0.718) and DFS (C-index: 0.700). The performance of the nomograms was verified in internal and external validations, which also implies the reliability of these nomograms. AJCC system is considered to be the benchmark for classifying patients with cancer and defining prognosis [53]. Compared with AJCC staging, the nomograms had higher C-index and net benefit, which implies the better clinical applicability of these nomograms.

To the best of our knowledge, this is the first attempt to construct a multinomial peripheral blood marker with all possible blood cell counts to identify high-risk populations with respect to recurrence or death in patients with CRC. Furthermore, this study has a large sample in both the training and external validation cohort. Our study also has several limitations. First, the PIIs were constructed and validated in two independent cohorts that included Chinese CRC patients. It would be better if multi-center validation can be carried out to verify whether the PIIs are universally applicable in other ethnicities. Second, our study found that the PIIs can independently and stably predict the prognosis of patients with stage II and stage III CRC. More studies should be conducted to evaluate whether the PIIs are also effective and feasible in patients with stage I and stage IV CRC. Third, our study was a retrospective cohort. Therefore it comes with a limitation that some data on clinicopathological characteristics are lacking, such as lymphovascular invasion, tumor budding, tumor-infiltrating lymphocyte, and microsatellite instability.

\section{Materials and Methods}

\subsection{Study Population}

A total of 11,127 primary stage II and III CRC patients confirmed by pathological diagnosis were enrolled in this study, including 4392 patients obtained from the Third Affiliated Hospital of Harbin Medical University, between January 2007 and December 2013 as the training cohort and 6735 patients obtained between January 2007 and December 2015 from the Fudan University Shanghai Cancer Center (FUSCC) as the external validation cohort. All these patients underwent radical resection surgery. A total of 1812 patients who met one or more of the following exclusion criteria were excluded (Figure 6): patients with age less than 18 years $(n=1)$; missing data on preoperative peripheral blood cell counts $(n=141)$; received neoadjuvant chemotherapy or other radiotherapy/chemotherapy before surgery ( $n=883)$; and patients lost to follow-up within 3 months $(n=787)$. Finally, 4154 and 5161 CRC patients were included in the training and validation cohort, respectively. This study complied with the standards of the Helsinki Declaration. Throughout this article, the term "prognostic marker" is defined according to REMARK Guidelines [54].

\subsection{Data Collection}

Data of patients' demographic and clinicopathological characteristics were obtained from retrospective medical records, including age, gender, BMI, history of hypertension and diabetes, tumor location, tumor diameter, pathological classification, differentiation degree, histologic classification, $\mathrm{T}$ and $\mathrm{N}$ stage, etc. Blood routine tests, which were based upon a single blood sample of each patient, were measured by an autoanalyzer (Sysmex XE-2100, Kobe, Japan). Data on peripheral blood cell counts including platelet, neutrophil, lymphocyte, monocyte, eosinophil, and basophil were extracted from the results of the first blood routine tests (limit to 30 days prior to surgery). 


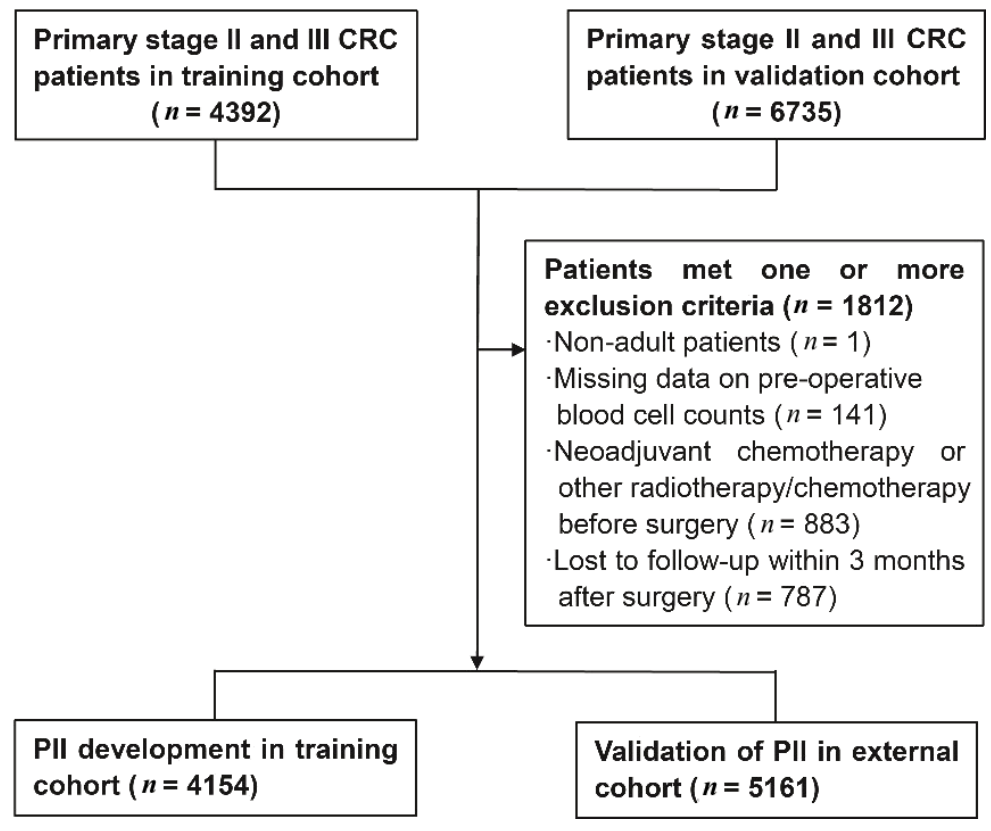

Figure 6. Flowchart of study patient selection.

Patients were followed up regularly in accordance with NCCN guidelines. The last time of follow-up for the training and external validation cohorts was 22 January 2019, and 18 July 2020, respectively. The survival information was obtained from the hospital medical records follow-up platform or contacts with patients by phone or email. OS was defined as the period from surgery to death from any cause, or the last contact. DFS was defined as the period from surgery to local recurrence, distant metastasis, a new primary tumor of CRC, or death, whichever comes first.

\subsection{Construction of the PII}

Prognostic factors of interest for constructing the PII were platelet, neutrophil, lymphocyte, monocyte, eosinophil, and basophil counts. The PII was constructed in the training cohort based on the following steps. First, RCS regression was performed to determine whether there was a nonlinear relationship between six types of blood cell counts and survival (OS and DFS). Factors that had significant nonlinear relationships with OS or DFS $(p<0.05)$ were converted into binary variables using X-tile 3.6.1 software [55] (Yale University, New Haven, CT, USA), while the others as continuous variables, without conversion. Second, univariate Cox proportional hazards models were implemented to investigate the association between the six types of blood cell counts and OS or DFS. Significant prognostic factors $(p<0.10)$ in the univariate analyses were then entered into multivariate Cox proportional hazards models. Finally, OS-PII and DFS-PII were constructed using blood cell counts associated with OS and DFS, with weights given by the corresponding coefficients from the multivariate Cox model.

\subsection{Statistical Analyses}

The missing data of included variables were filled in by using the multiple imputation method [56]. Student's $t$-tests for normally distributed continuous variables, $\chi^{2}$ tests for categorical variables, and Mann-Whitney $U$ tests for non-normally distributed continuous variables were used to evaluate the differences between training and validation cohorts. X-tile 3.6.1 software [55] was used to determine the optimal cut-off values for the PIIs in the training cohort, which were also used in the external validation cohort. The $\chi^{2}$ tests were performed to assess the association between the PIIs and clinicopathological characteristics. The 1-, 3-, 5- and 10-year OS and DFS were calculated using the Kaplan-Meier method, and the difference between two PII levels was compared using log-rank tests. The prognostic 
value of clinicopathological characteristics and PIIs were estimated using univariate and multivariate Cox analyses. The results of Cox analyses were presented as $\mathrm{HR}$ and $95 \% \mathrm{CI}$. Additionally, subgroup analyses were also conducted, stratified by age ( $<60$ years old; $\geq 60$ years old), gender (male; female), tumor location (colon; rectum), tumor diameter ( $<50 \mathrm{~mm}$; $\geq 50 \mathrm{~mm}), \mathrm{CA} 19-9(<37 \mathrm{U} / \mathrm{mL} ; \geq 37 \mathrm{U} / \mathrm{mL})$, and postoperative chemotherapy (no or yes).

The nomograms for possible prognostic factors associated with OS and DFS were developed to predict the probability of 1-, 3- and 5-year survival recurrence/metastasis for CRC patients. Multivariate Cox analyses determined the effects of prognostic factors on a nomogram, and only the factors with a $p$-value $<0.05$ were finally incorporated into the nomogram. The prediction accuracy of the nomograms was evaluated by the C-index [50,57]. The value of the C-index ranged from 1.0 (perfect concordance) to 0.0 (perfect discordance), and a value of 0.5 is equivalent to a random chance. Nomograms map the predicted probabilities into points on a scale from 0 to 100, and can be interpreted by accumulating the points correspond to the predicted probability, which is indicated at the top of the scale [57]. Bootstrapping techniques were used for internal validation of the prognostic models, and the calibration of nomograms was assessed graphically by plotting the actual probabilities versus the nomogram-predicted probabilities $[50,57]$. Decision curve analyses were also performed for the prognostic models of nomograms and AJCC system. The net benefits of nomograms and AJCC system were compared to evaluate the clinical applicability of these two models. Time-dependent ROC analyses were performed, and the estimated AUCs were calculated to compare the prognostic abilities of the PIIs, TNM staging, their combination, and biomarkers previously reported (NLR, PLR, LMR, and SII) [58,59].

All statistical analyses were performed with SPSS 24.0 (SPSS Inc., Chicago, IL, USA) and R 3.6.2 software (Institute for Statistics and Mathematics, Vienna, Austria). Two sided $p<0.05$ was considered statistically significant.

\section{Conclusions}

We constructed a novel PII by integrating all the preoperative peripheral blood cell counts associated with prognosis and systematically analyzed the role of PIIs in the prognosis of CRC. Our study demonstrates that both OS-PII and DFS-PII are independent factors for predicting the prognosis of CRC and could enhance the prognostic ability of TNM staging by combination. The nomograms based on OS and DFS can be recommended for the personalized survival prediction of patients with CRC.

Supplementary Materials: The following are available online at https:/ / www.mdpi.com/2072-669 4/13/1/3/s1, Figure S1: Restricted cubic spline regression for assessing the nonlinear relationship between preoperative peripheral blood cell counts and overall survival of patients with colorectal cancer, Figure S2: Restricted cubic spline regression for assessing the nonlinear relationship between preoperative peripheral blood cell counts and disease-free survival of patients with colorectal cancer, Figure S3: Determination of the cut-off value for platelet, lymphocyte, and eosinophil counts, Figure S4: Determination of the optimal cut-off value for the OS-PII and DFS-PII, Figure S5: Risk stratification combining PIIs and TNM staging in relation to overall survival and disease-free survival of colorectal cancer in the validation cohort, Figure S6. Subgroup analyses for the association of OS-PII and DFS-PII with the prognosis of colorectal cancer in the training cohort, Figure S7: Subgroup analyses for the association of OS-PII and DFS-PII with the prognosis of colorectal cancer in the validation cohort, Figure S8: The time-dependent AUCs of PIIs, TNM staging, a combination of PIIs and TNM staging, NLR, PLR, LMR, and SII in the training cohort, Figure S9: Decision curve analysis for 5-year overall survival prediction (A) and 5-year disease-free survival prediction (B) in the training cohort, Figure S10: Decision curve analysis for 5-year overall survival prediction (A) and 5-year disease-free survival prediction (B) in the validation cohort, Table S1: The prognostic effects of six types of preoperative blood cell count for patients with colorectal cancer in the training cohort, Table S2: Association of OS-PII and DFS-PII with clinicopathological characteristics of colorectal cancer in the training cohort, Table S3: Association of OS-PII and DFS-PII with clinicopathological characteristics of colorectal cancer in the validation cohort, Table S4: The overall survival rates at 
1-, 3-, 5- and 10-year in groups stratified by OS-PII in the training cohort, Table S5: The diseasefree survival rates at 1-, 3-, 5- and 10-year in groups stratified by DFS-PII in the training cohort, Table S6: The overall survival rates at 1-, 3-, 5-year in groups stratified by OS-PII in the validation cohort, Table S7: The disease-free survival rates at 1-, 3-, 5-year in groups stratified by DFS-PII in the validation cohort, Table S8: Time-dependent ROC analyses for predicting overall survival of patients with colorectal cancer in the training cohort, Table S9: Time-dependent ROC analyses for predicting disease-free survival of patients with colorectal cancer in the training cohort, Table S10: Time-dependent ROC analyses for predicting overall survival of patients with colorectal cancer in the validation cohort, Table S11: Time-dependent ROC analyses for predicting disease-free survival of patients with colorectal cancer in the validation cohort, Table S12: The C-index of AJCC system and nomograms, Table S13: Time-dependent ROC analyses stratified by TNM staging for predicting survival of patients with colorectal cancer in the training cohort, Table S14: Time-dependent ROC analyses stratified by TNM staging for predicting survival of patients with colorectal cancer in the validation cohort.

Author Contributions: J.F., Y.Z. (Yashuang Zhao), Y.L. (Yanlong Liu), and J.Z. had full access to all of the data in the study and takes responsibility for the integrity of the data and accuracy of the data analysis. Conceptualization, J.F., S.C., J.Z., Y.L. (Yanlong Liu) and Y.Z. (Yashuang Zhao); Data curation, J.F., F.D., L.Z. (Lijie Zhang), T.T., Y.L. (Yupeng Liu), L.Z. (Lei Zhang), Y.L. (Ying Liu), Y.Z. (Yuanyuan Zhang), J.X., S.M., C.J., S.S., X.L., L.Z. (Liyuan Zhao), D.Z., L.K., L.G. and T.Z.; Formal analysis, J.F., D.L., H.H. and L.Z. (Lei Zhang); Investigation, F.D. and L.Z. (Lijie Zhang); Supervision, J.Z.; Writing-original draft, J.F.; Writing-review \& editing, J.F., D.L., Y.L. (Yanlong Liu) and Y.Z. (Yashuang Zhao). All authors have read and agreed to the published version of the manuscript.

Funding: This research received no external funding.

Conflicts of Interest: The authors declare no conflict of interest.

\section{References}

1. Bray, F.; Ferlay, J.; Soerjomataram, I.; Siegel, R.L.; Torre, L.A.; Jemal, A. Global cancer statistics 2018: GLOBOCAN estimates of incidence and mortality worldwide for 36 cancers in 185 countries. CA Cancer J. Clin. 2018, 68, 394-424. [CrossRef] [PubMed]

2. Chen, W.; Zheng, R.; Baade, P.D.; Zhang, S.; Zeng, H.; Bray, F.; Jemal, A.; Yu, X.Q.; He, J. Cancer statistics in China, 2015. CA Cancer J. Clin. 2016, 66, 115-132. [CrossRef] [PubMed]

3. Arnold, M.; Sierra, M.S.; Laversanne, M.; Soerjomataram, I.; Jemal, A.; Bray, F. Global patterns and trends in colorectal cancer incidence and mortality. Gut 2017, 66, 683-691. [CrossRef] [PubMed]

4. DeSantis, C.E.; Lin, C.C.; Mariotto, A.B.; Siegel, R.L.; Stein, K.D.; Kramer, J.L.; Alteri, R.; Robbins, A.S.; Jemal, A. Cancer treatment and survivorship statistics, 2014. CA Cancer J. Clin. 2014, 64, 252-271. [CrossRef] [PubMed]

5. Le Voyer, T.E.; Sigurdson, E.R.; Hanlon, A.L.; Mayer, R.J.; Macdonald, J.S.; Catalano, P.J.; Haller, D.G. Colon cancer survival is associated with increasing number of lymph nodes analyzed: A secondary survey of intergroup trial INT-0089. J. Clin. Oncol. 2003, 21, 2912-2919. [CrossRef] [PubMed]

6. O'Connell, J.B.; Maggard, M.A.; Ko, C.Y. Colon cancer survival rates with the new American Joint Committee on Cancer sixth edition staging. J. Natl. Cancer Inst. 2004, 96, 1420-1425. [CrossRef] [PubMed]

7. Morris, M.; Platell, C.; de Boer, B.; McCaul, K.; Iacopetta, B. Population-based study of prognostic factors in stage II colonic cancer. Br. J. Surg. 2006, 93, 866-871. [CrossRef]

8. Karapetis, C.S.; Khambata-Ford, S.; Jonker, D.J.; O'Callaghan, C.J.; Tu, D.; Tebbutt, N.C.; Simes, R.J.; Chalchal, H.; Shapiro, J.D.; Robitaille, S.; et al. K-ras mutations and benefit from cetuximab in advanced colorectal cancer. N. Engl. J. Med. 2008, 359, 1757-1765. [CrossRef]

9. Tol, J.; Nagtegaal, I.D.; Punt, C.J. BRAF mutation in metastatic colorectal cancer. N. Engl. J. Med. 2009, 361, 98-99. [CrossRef]

10. Roth, A.D.; Tejpar, S.; Delorenzi, M.; Yan, P.; Fiocca, R.; Klingbiel, D.; Dietrich, D.; Biesmans, B.; Bodoky, G.; Barone, C.; et al. Prognostic role of KRAS and BRAF in stage II and III resected colon cancer: Results of the translational study on the PETACC-3, EORTC 40993, SAKK 60-00 trial. J. Clin. Oncol 2010, 28, 466-474. [CrossRef]

11. Balkwill, F.; Mantovani, A. Inflammation and cancer: Back to Virchow? Lancet 2001, 357, 539-545. [CrossRef]

12. Hanahan, D.; Weinberg, R.A. Hallmarks of cancer: The next generation. Cell 2011, 144, 646-674. [CrossRef] [PubMed]

13. Greten, F.R.; Grivennikov, S.I. Inflammation and Cancer: Triggers, Mechanisms, and Consequences. Immunity 2019, 51, 27-41. [CrossRef] [PubMed]

14. Trinchieri, G. Cancer and inflammation: An old intuition with rapidly evolving new concepts. Annu. Rev. Immunol. 2012, 30, 677-706. [CrossRef] [PubMed]

15. Mantovani, A.; Allavena, P.; Sica, A.; Balkwill, F. Cancer-related inflammation. Nature 2008, 454, 436-444. [CrossRef] [PubMed] 
16. Portale, G.; Cavallin, F.; Valdegamberi, A.; Frigo, F.; Fiscon, V. Platelet-to-Lymphocyte Ratio and Neutrophil-to-Lymphocyte Ratio Are Not Prognostic Biomarkers in Rectal Cancer Patients with Curative Resection. J. Gastrointest. Surg. 2018, 22, 1611-1618. [CrossRef]

17. Li, Z.; Zhao, R.; Cui, Y.; Zhou, Y.; Wu, X. The dynamic change of neutrophil to lymphocyte ratio can predict clinical outcome in stage I-III colon cancer. Sci. Rep. 2018, 8, 9453. [CrossRef]

18. Kim, J.H.; Lee, J.Y.; Kim, H.K.; Lee, J.W.; Jung, S.G.; Jung, K.; Kim, S.E.; Moon, W.; Park, M.I.; Park, S.J. Prognostic significance of the neutrophil-to-lymphocyte ratio and platelet-to-lymphocyte ratio in patients with stage III and IV colorectal cancer. World J. Gastroenterol. 2017, 23, 505-515. [CrossRef]

19. Wu, Q.B.; Wang, M.; Hu, T.; He, W.B.; Wang, Z.Q. Prognostic role of the lymphocyte-to-monocyte ratio in patients undergoing resection for nonmetastatic rectal cancer. Medicine (Baltimore) 2016, 95, e4945. [CrossRef]

20. Mahsuni Sevinc, M.; Riza Gunduz, U.; Kinaci, E.; Armagan Aydin, A.; Bayrak, S.; Umar Gursu, R.; Gunduz, S. Preoperative neutrophil-to-lymphocyte ratio and plateletto- lymphocyte ratio as new prognostic factors for patients with colorectal cancer. J. Buon 2016, 21, 1153-1157.

21. Ozawa, T.; Ishihara, S.; Nishikawa, T.; Tanaka, T.; Tanaka, J.; Kiyomatsu, T.; Hata, K.; Kawai, K.; Nozawa, H.; Kazama, S.; et al. The preoperative platelet to lymphocyte ratio is a prognostic marker in patients with stage II colorectal cancer. Int. J. Colorectal Dis. 2015, 30, 1165-1171. [CrossRef] [PubMed]

22. Szkandera, J.; Pichler, M.; Absenger, G.; Stotz, M.; Arminger, F.; Weissmueller, M.; Schaberl-Moser, R.; Samonigg, H.; Kornprat, P.; Stojakovic, T.; et al. The elevated preoperative platelet to lymphocyte ratio predicts decreased time to recurrence in colon cancer patients. Am. J. Surg. 2014, 208, 210-214. [CrossRef] [PubMed]

23. Malietzis, G.; Giacometti, M.; Askari, A.; Nachiappan, S.; Kennedy, R.H.; Faiz, O.D.; Aziz, O.; Jenkins, J.T. A preoperative neutrophil to lymphocyte ratio of 3 predicts disease-free survival after curative elective colorectal cancer surgery. Ann. Surg. 2014, 260, 287-292. [CrossRef] [PubMed]

24. Wei, Y.; Zhang, X.; Wang, G.; Zhou, Y.; Luo, M.; Wang, S.; Hong, C. The impacts of pretreatment circulating eosinophils and basophils on prognosis of stage-colorectal cancer. Asia Pac. J. Clin. Oncol. 2018, 14, e243-e251. [CrossRef]

25. Sertoglu, E.; Uyanik, M.; Kayadibi, H. Value of neutrophil to lymphocyte ratio as a biomarker in colorectal adenocarcinoma. Eur. J. Cancer Prev. 2016, 25, 1-2. [CrossRef]

26. Marx, J. Cancer research. Inflammation and cancer: The link grows stronger. Science 2004, 306, 966-968. [CrossRef]

27. Kuraishy, A.; Karin, M.; Grivennikov, S.I. Tumor promotion via injury- and death-induced inflammation. Immunity 2011, 35, 467-477. [CrossRef]

28. Grivennikov, S.I.; Greten, F.R.; Karin, M. Immunity, inflammation, and cancer. Cell 2010, 140, 883-899. [CrossRef]

29. Zhan, X.; Sun, X.; Hong, Y.; Wang, Y.; Ding, K. Combined Detection of Preoperative Neutrophil-to-Lymphocyte Ratio and CEA as an Independent Prognostic Factor in Nonmetastatic Patients Undergoing Colorectal Cancer Resection Is Superior to NLR or CEA Alone. Biomed. Res. Int. 2017, 2017, 3809464. [CrossRef]

30. Guthrie, G.J.; Roxburgh, C.S.; Farhan-Alanie, O.M.; Horgan, P.G.; McMillan, D.C. Comparison of the prognostic value of longitudinal measurements of systemic inflammation in patients undergoing curative resection of colorectal cancer. Br. J. Cancer 2013, 109, 24-28. [CrossRef]

31. Shibutani, M.; Maeda, K.; Nagahara, H.; Noda, E.; Ohtani, H.; Nishiguchi, Y.; Hirakawa, K. A high preoperative neutrophilto-lymphocyte ratio is associated with poor survival in patients with colorectal cancer. Anticancer Res. 2013, 33, 3291-3294. [PubMed]

32. Kubo, T.; Ono, S.; Ueno, H.; Shinto, E.; Yamamoto, J.; Hase, K. Impact of the perioperative neutrophil-to-lymphocyte ratio on the long-term survival following an elective resection of colorectal carcinoma. Int. J. Colorectal Dis. 2014, 29, 1091-1099. [CrossRef] [PubMed]

33. He, W.; Yin, C.; Guo, G.; Jiang, C.; Wang, F.; Qiu, H.; Chen, X.; Rong, R.; Zhang, B.; Xia, L. Initial neutrophil lymphocyte ratio is superior to platelet lymphocyte ratio as an adverse prognostic and predictive factor in metastatic colorectal cancer. Med. Oncol. 2013, 30, 439. [CrossRef] [PubMed]

34. Galizia, G.; Lieto, E.; Zamboli, A.; De Vita, F.; Castellano, P.; Romano, C.; Auricchio, A.; Cardella, F.; De Stefano, L.; Orditura, M. Neutrophil to lymphocyte ratio is a strong predictor of tumor recurrence in early colon cancers: A propensity score-matched analysis. Surgery 2015, 158, 112-120. [CrossRef]

35. Walsh, S.R.; Cook, E.J.; Goulder, F.; Justin, T.A.; Keeling, N.J. Neutrophil-lymphocyte ratio as a prognostic factor in colorectal cancer. J. Surg. Oncol. 2005, 91, 181-184. [CrossRef]

36. Absenger, G.; Szkandera, J.; Stotz, M.; Postlmayr, U.; Pichler, M.; Ress, A.L.; Schaberl-Moser, R.; Loibner, H.; Samonigg, H.; Gerger, A. Preoperative neutrophil-to-lymphocyte ratio predicts clinical outcome in patients with stage II and III colon cancer. Anticancer Res. 2013, 33, 4591-4594.

37. Ying, H.Q.; Deng, Q.W.; He, B.S.; Pan, Y.Q.; Wang, F.; Sun, H.L.; Chen, J.; Liu, X.; Wang, S.K. The prognostic value of preoperative NLR, d-NLR, PLR and LMR for predicting clinical outcome in surgical colorectal cancer patients. Med. Oncol. 2014, $31,305$. [CrossRef]

38. Gijsbers, K.; Gouwy, M.; Struyf, S.; Wuyts, A.; Proost, P.; Opdenakker, G.; Penninckx, F.; Ectors, N.; Geboes, K.; Van Damme, J. GCP-2/CXCL6 synergizes with other endothelial cell-derived chemokines in neutrophil mobilization and is associated with angiogenesis in gastrointestinal tumors. Exp. Cell Res. 2005, 303, 331-342. [CrossRef] 
39. Mantovani, A.; Cassatella, M.A.; Costantini, C.; Jaillon, S. Neutrophils in the activation and regulation of innate and adaptive immunity. Nat. Rev. Immunol. 2011, 11, 519-531. [CrossRef]

40. Mizuno, R.; Kawada, K.; Itatani, Y.; Ogawa, R.; Kiyasu, Y.; Sakai, Y. The Role of Tumor-Associated Neutrophils in Colorectal Cancer. Int. J. Mol. Sci. 2019, 20, 529. [CrossRef]

41. Augier, S.; Ciucci, T.; Luci, C.; Carle, G.F.; Blin-Wakkach, C.; Wakkach, A. Inflammatory blood monocytes contribute to tumor development and represent a privileged target to improve host immunosurveillance. J. Immunol. 2010, 185, 7165-7173. [CrossRef] [PubMed]

42. Condeelis, J.; Pollard, J.W. Macrophages: Obligate partners for tumor cell migration, invasion, and metastasis. Cell 2006, 124, 263-266. [CrossRef] [PubMed]

43. Diakos, C.I.; Charles, K.A.; McMillan, D.C.; Clarke, S.J. Cancer-related inflammation and treatment effectiveness. Lancet Oncol. 2014, 15, e493-e503. [CrossRef]

44. Gaertner, F.; Massberg, S. Patrolling the vascular borders: Platelets in immunity to infection and cancer. Nat. Rev. Immunol. 2019, 19, 747-760. [CrossRef]

45. Michael, J.V.; Wurtzel, J.G.T.; Mao, G.F.; Rao, A.K.; Kolpakov, M.A.; Sabri, A.; Hoffman, N.E.; Rajan, S.; Tomar, D.; Madesh, M.; et al. Platelet microparticles infiltrating solid tumors transfer miRNAs that suppress tumor growth. Blood 2017, 130, 567-580. [CrossRef]

46. Prizment, A.E.; Vierkant, R.A.; Smyrk, T.C.; Tillmans, L.S.; Lee, J.J.; Sriramarao, P.; Nelson, H.H.; Lynch, C.F.; Thibodeau, S.N.; Church, T.R.; et al. Tumor eosinophil infiltration and improved survival of colorectal cancer patients: Iowa Women's Health Study. Mod. Pathol. 2016, 29, 516-527. [CrossRef]

47. Xie, F.; Liu, L.B.; Shang, W.Q.; Chang, K.K.; Meng, Y.H.; Mei, J.; Yu, J.J.; Li, D.J.; Li, M.Q. The infiltration and functional regulation of eosinophils induced by TSLP promote the proliferation of cervical cancer cell. Cancer Lett. 2015, 364, 106-117. [CrossRef]

48. Kozak, M.M.; von Eyben, R.; Pai, J.S.; Anderson, E.M.; Welton, M.L.; Shelton, A.A.; Kin, C.; Koong, A.C.; Chang, D.T. The Prognostic Significance of Pretreatment Hematologic Parameters in Patients Undergoing Resection for Colorectal Cancer. Am. J. Clin. Oncol. 2017, 40, 405-412. [CrossRef]

49. Brenner, H.; Kloor, M.; Pox, C.P. Colorectal cancer. Lancet 2014, 383, 1490-1502. [CrossRef]

50. Balachandran, V.P.; Gonen, M.; Smith, J.J.; DeMatteo, R.P. Nomograms in oncology: More than meets the eye. Lancet Oncol. 2015, 16, e173-e180. [CrossRef]

51. Sternberg, C.N. Are nomograms better than currently available stage groupings for bladder cancer? J. Clin. Oncol. 2006, 24, 3819-3820. [CrossRef] [PubMed]

52. Touijer, K.; Scardino, P.T. Nomograms for staging, prognosis, and predicting treatment outcomes. Cancer 2009, 115, 3107-3111 [CrossRef] [PubMed]

53. Amin, M.B.; Greene, F.L.; Edge, S.B.; Compton, C.C.; Gershenwald, J.E.; Brookland, R.K.; Meyer, L.; Gress, D.M.; Byrd, D.R.; Winchester, D.P. The Eighth Edition AJCC Cancer Staging Manual: Continuing to build a bridge from a population-based to a more "personalized" approach to cancer staging. CA Cancer J. Clin. 2017, 67, 93-99. [CrossRef] [PubMed]

54. McShane, L.M.; Altman, D.G.; Sauerbrei, W.; Taube, S.E.; Gion, M.; Clark, G.M. Reporting recommendations for tumor marker prognostic studies (REMARK). J. Natl. Cancer Inst. 2005, 97, 1180-1184. [CrossRef] [PubMed]

55. Camp, R.L.; Dolled-Filhart, M.; Rimm, D.L. X-tile: A new bio-informatics tool for biomarker assessment and outcome-based cut-point optimization. Clin. Cancer Res. 2004, 10, 7252-7259. [CrossRef]

56. Sterne, J.A.; White, I.R.; Carlin, J.B.; Spratt, M.; Royston, P.; Kenward, M.G.; Wood, A.M.; Carpenter, J.R. Multiple imputation for missing data in epidemiological and clinical research: Potential and pitfalls. BMJ 2009, 338, b2393. [CrossRef]

57. Iasonos, A.; Schrag, D.; Raj, G.V.; Panageas, K.S. How to build and interpret a nomogram for cancer prognosis. J. Clin. Oncol. 2008, 26, 1364-1370. [CrossRef]

58. Kamarudin, A.N.; Cox, T.; Kolamunnage-Dona, R. Time-dependent ROC curve analysis in medical research: Current methods and applications. BMC Med. Res. Methodol. 2017, 17, 53. [CrossRef]

59. Heagerty, P.J.; Lumley, T.; Pepe, M.S. Time-dependent ROC curves for censored survival data and a diagnostic marker. Biometrics 2000, 56, 337-344. [CrossRef] 